Series A

I. MATHEMATICA

393

\title{
QUASICONFORMAL MAPS IN MANIFOLDS
}

BY

KALEVI SUOMINEN

H E L S I N I 1966

S U O M A L A INEN TIEDEAKATEMIA

doi:10.5186/aasfm.1967.393 
Communicated 13 May 1966 by OLli Lehto and K. I. Virtanen 


\section{Preface}

I am deeply indebted to Professor Olli Lehto and Professor K. I. Virtanen for suggesting this subject and for their kind interest and valuable advice.

I also wish to thank the Niilo Helander Foundation for its financial support.

Helsinki, May 1966.

Kalevi Suominen 


\section{Introduction}

It is well-known that each 2-dimensional Riemannian manifold is locally conformally equivalent to the plane. Hence, in the 2-dimensional case, the local properties of quasiconformal maps may be studied in the plane. In higher dimensions the situation is different. There is no reason for disregarding Riemannian manifolds other than the Euclidean space. In the first part of this paper we develop and study concepts which are needed for the theory of quasiconformal maps in Riemannian manifolds. As an application, we generalize in the second part certain results of Teichmüller, Wittich and Belinskij (cf. Lehto-Virtanen [1], p. 233).

\section{Notations}

The tangent bundle of a differentiable manifold $M$ is denoted by $T M$. The derivative of a differentiable map $f: M \rightarrow N$ is a fibre map $D f$ : $T M \rightarrow T N$. We denote by int $M$ the complement of the boundary of $M$.

Let $M$ be a $C^{p}$-manifold. An open set $U \subset M$ is called a $C^{q}$-coordinate neighbourhood of $M, q \leq p$, if there is a $C^{q}$-diffeomorphism of $U$ onto an open set in $\mathbf{R}^{n}$.

The support of a function $\varphi: M \rightarrow \mathbf{R}$, i.e. the closure of $\{x \in M \mid$ $\varphi(x) \neq 0\}$, is denoted by $\operatorname{supp} \varphi$.

$\mathbf{R}$ : the set of real numbers.

$\mathbf{R}^{+}$: the set of positive real numbers.

$\mathbf{R}_{+}^{n}$ : the set $\left\{x=\left(x_{n}, \ldots, x_{n}\right) \in \mathbf{R}^{n} \mid x_{n}>0\right\}$.

$B^{n}(x, r)=\left\{y \in \mathbf{R}^{n}|| y-x \mid<r\right\}$.

$S^{n-1}(x, r)=\left\{y \in \mathbf{R}^{n}|| y-x \mid=r\right\}$.

$\Omega_{n}$ : the measure of $B^{n}(0,1)$.

$\omega_{n}$ : the measure of $S^{n-1}(0,1)$.

If there is no danger of confusion, we may omit the index $n$ in $B^{n}$ or $S^{n}$. Similarly, $x$ may be omitted if $x=0$ and $r$ if $r=1$.

A Borel measure in a manifold $M$ is a measure which is defined in the family of Borel sets of $M$. 


\section{Part I}

In the first part of the paper we develop the concepts which are necessary for the theory of quasiconformal maps in manifolds. To some extent this consists of generalizing the definitions given for the euclidean local case and stating simple results which are not readily accessible in the literature. For some notions, however, marked differences appear by departure from the classical case. This occurs e.g. in sections 6 and 8 where $A C L^{p}$-maps and dilatations are considered. In the whole, we have not tried to confine the discussion to the minimum and have in some places presented more material than that which would be absolutely indispensable for our purposes.

\section{Riemannian manifolds}

In this section we introduce some terminology and assumptions about manifolds.

1.1. Theorem. Let $M$ be a manifold satisfying the following conditions:

a) $M$ is infinitely differentiable,

b) $M$ is paracompact,

c) $M$ is connected.

Then $M$ has the following properties:

d) $M$ admits partitions of unity of class $C^{\infty}$,

e) $M$ has a countable base for open sets,

f) $M$ admits a Riemannian metric,

g) $M$ is metrizable.

For the terminology we refer to the sources given below.

Proof. The property d) is proved in Lang [1], p. 30. A proof for e) may be given following the argument in Bourbaki [2], pp. 111-112, and using c) in addition to b). A Riemannian metric is constructed in a well-known manner by means of partitions of unity (Lang [1], p. 98). Finally, metrizability follows e.g. by Urysohn's theorem (Kelley [1], p. 125).

1.2. Convention. In the sequel all manifolds are assumed to satisfy the conditions a) $-\mathrm{g}$ ) of Theorem 1.1. By a Riemannian manifold we mean one which has a (fixed) Riemannian metric. A submanifold of a Riemannian manifold $M$ is called a Riemannian submanifold if its Riemannian metric is induced by the Riemannian metric of $M$.

Manifolds with boundary are also admitted. However, if products of manifolds are considered, it is tacitly assumed that at most one of the factors has a non-empty boundary. 


\section{Measurability}

Before developing a theory of measure for Riemannian manifolds we study first questions of measurability. Since one of our basic requirements is that continuous maps preserve measurability, it is natural to consider Borel sets. The family of Borel sets in a topological space is defined in the ordinary way as the smallest $\sigma$-algebra containing all open sets.

2.1. Definition. Let $M$ and $N$ be manifolds. A map $f: M \rightarrow N$ is measurable if the preimage $f^{-1}(U)$ of each open set $U \subset N$ is a Borel set. In particular, every continuous map is measurable. A measurable realvalued map $f: M \rightarrow \mathbf{R}$ is also called a Baire function.

2.2. Lemma. Let $M$ and $N$ be manifolds and $f: M \rightarrow N$ a measurable map. Then the preimage $f^{-1}(E)$ of each Borel set $E \subset N$ is a Borel set. If $g: N \rightarrow L$ is a measurable map, then $g \circ f: M \rightarrow L$ is also measurable.

Proof: The family of subsets $E$ of $N$, such that $f^{-1}(E)$ is a Borel set, is a $\sigma$-algebra. Since it contains every open set $U \subset N$, it also contains all Borel sets of $N$.

The second assertion is an immediate consequence of the first one.

2.3. Lemma. Let $M, N$ and $N^{\prime}$ be manifolds, $f: M \rightarrow N$ and $f^{\prime}: M \rightarrow N^{\prime}$ maps. Then the product map $g: M \rightarrow N \times N^{\prime}$ is measurable if and only if $f$ and $f^{\prime}$ are measurable.

Proof: The canonical projections $p: N \times N^{\prime} \rightarrow N$ and $p^{\prime}: N \times N^{\prime} \rightarrow N^{\prime}$ are continuous, hence measurable. Thus the measurability of $g$ implies that $f=p \circ g$ and $f^{\prime}=p^{\prime} \circ g$ are measurable by Lemma 2.2. On the other hand, if $f$ and $f^{\prime}$ are measurable, then $g^{-1}\left(U \times U^{\prime}\right)=f^{-1}(U) \cap f^{\prime-1}\left(U^{\prime}\right)$ is a Borel set for all open sets $U \subset N^{\prime}$ and $U^{\prime} \subset N^{\prime}$. Since the products $U \times U^{\prime}$ form a base for open sets in $N^{\prime} \times N^{\prime}, g$ is measurable.

2.4. Lemma. Let $M$ be a manifold and $E$ a finite dimensional real vector space. If $f: M \rightarrow E$ and $g: M \rightarrow E$ are measurable maps, then $f+g$ : $M \rightarrow E$ is measurable, and $\lambda f: M \rightarrow E$ is measurable for each Barre function $\lambda$ in $M$.

Proof: The addition $E \times E \rightarrow E$ is continuous. Hence the measurability of $f+g$ follows from Lemmas 2.3 and 2.2. The proof is similar for $\lambda f$.

For the next lemma we recall that the set of linear maps $\mathbf{R}^{n} \rightarrow \mathbf{R}^{m}$ may be canonically represented by matrices of type $(m, n)$ with real entries. Hence to each map $M \rightarrow L\left(\mathbf{R}^{n}, \mathbf{R}^{m}\right)$ corresponds a matrix function $\left(\lambda_{i j}\right), \quad \lambda_{i j}: M \rightarrow \mathbf{R}, i=1, \ldots, m, j=1, \ldots, n$.

2.5. Lemma. Let $M$ be a manifold. Let $f: M \rightarrow L\left(\mathbf{R}^{n}, \mathbf{R}^{m}\right)$ be a map and $\left(\lambda_{i j}\right), i=1, \ldots, m, j=1, \ldots, n$, the corresponding matrix function. Then the following conditions are equivalent:

a) $f$ is measurable,

b) the functions $\lambda_{i j}$ are measurable, 
c) the map $g: M \times \mathbf{R}^{n} \rightarrow \mathbf{R}^{m}$ defined by

$$
g(x, v)=f(x)(v), \quad x \in M, v \in \mathbf{R}^{n},
$$

is measurable.

Proof: The evaluation map $e: L\left(\mathbf{R}^{n}, \mathbf{R}^{m}\right) \times \mathbf{R}^{n} \rightarrow \mathbf{R}^{m}$, defined by

$$
e(u, v)=u(v), \quad u \in L\left(\mathbf{R}^{n}, \mathbf{R}^{m}\right), \quad v \in \mathbf{R}^{n},
$$

is continuous. Hence a) implies c) by Lemmas 2.3 and 2.2.

Let $e_{j} \in \mathbf{R}^{n}, j=1, \ldots, n$, denote the $j$-th canonical basis vector $(0, \ldots, 1, \ldots, 0)$. If $\mathrm{c})$ holds, then the maps $f_{j}: M \rightarrow \mathbf{R}^{m}$, defined by $f_{j}(x)=g\left(x, e_{j}\right)$, are measurable. On the other hand $f_{j}=\lambda_{1 j} \times \ldots \times \lambda_{m j}$. Thus c) implies b).

Finally, b) implies a), since $f$ may be regarded as the cartesian product of the maps $\lambda_{i j}$.

2.6. Definition. Let $M$ be a manifold. A set $E \subset M$ is a null set, if $\varphi(E \cap U)$ has Lebesgue measure zero for each coordinate neighbourhood $U \subset M$ and each coordinate map $\varphi: U \rightarrow \mathbf{R}^{n}$ (of class $C^{\infty}$ ).

A condition is said to hold for almost every $x \in M$, or almost everywhere in $M$, if it holds everywhere except in a null set.

2.7. Remark. Since sets of Lebesgue measure zero in $\mathbf{R}^{n}$ are preserved by $C^{1}$-diffeomorphisms, the coordinate maps $\varphi$ may be of class $C^{\mathbf{1}}$. In addition, only coordinate neighbourhoods $U$ belonging to a fixed cover $\left(U_{i}\right)$ of $M$ need be taken into consideration. We also remark that since $\left(U_{i}\right)$ may be chosen to be countable (Theorem 1.1. e)), the definition will agree with the measure defined later in section 3 .

2.8. Convention. As derivatives of functions differentiable almost everywhere we shall meet functions which are not defined in a Borel null set. If such a function is measurable in its set of definition its extension by a constant value will also be measurable. We agree to carry out always such an extension by the value 0 . Hence we may regard all functions as defined everywhere.

2.9. Lemma. Let $M$ and $N$ be Riemannian manifolds, $f: M \rightarrow N$ a continuous map, and $u: T M \rightarrow T N$ a measurable fibre map over $f$ (i.e. $u$ maps each fibre $T_{x} M, x \in M$, linearly into the fibre $\left.T_{f(x)} N\right)$. Then $\|u\|$ and $\operatorname{det} u$ are Baire functions in $M$.

Proof: In case $M=U$ and $N=V$ are open sets in $\mathbf{R}^{n}$ and $\mathbf{R}^{m}$ we may represent $u$ by a measurable map $g: U \rightarrow L\left(\mathbf{R}^{n}, \mathbf{R}^{m}\right.$ ) (Lemmas 2.3 and 2.5). The norm and the determinant are continuous functions in $L\left(\mathbf{R}^{n}, \mathbf{R}^{m}\right)$ which depend continuously on parameter points $x \in U$ and $y=f(x) \in V$. Hence the assertion follows by Lemma 2.2. Since the question is local, this also proves the general case. 


\section{Measurability}

Before developing a theory of measure for Riemannian manifolds we study first questions of measurability. Since one of our basic requirements is that continuous maps preserve measurability, it is natural to consider Borel sets. The family of Borel sets in a topological space is defined in the ordinary way as the smallest $\sigma$-algebra containing all open sets.

2.1. Definition. Let $M$ and $N$ be manifolds. A map $f: M \rightarrow N$ is measurable if the preimage $f^{-1}(U)$ of each open set $U \subset N$ is a Borel set. In particular, every continuous map is measurable. A measurable realvalued map $f: M \rightarrow \mathbf{R}$ is also called a Baire function.

2.2. Lemma. Let $M$ and $N$ be manifolds and $f: M \rightarrow N$ a measurable map. Then the preimage $f^{-1}(E)$ of each Borel set $E \subset N$ is a Borel set. If $g: N \rightarrow L$ is a measurable map, then $g \circ f: M \rightarrow L$ is also measurable.

Proof: The family of subsets $E$ of $N$, such that $f^{-1}(E)$ is a Borel set, is a $\sigma$-algebra. Since it contains every open set $U \subset N$, it also contains all Borel sets of $N$.

The second assertion is an immediate consequence of the first one.

2.3. Lemma. Let $M, N$ and $N^{\prime}$ be manifolds, $f: M \rightarrow N$ and $f^{\prime}: M \rightarrow N^{\prime}$ maps. Then the product map $g: M \rightarrow N \times N^{\prime}$ is measurable if and only if $f$ and $f^{\prime}$ are measurable.

Proof: The canonical projections $p: N \times N^{\prime} \rightarrow N$ and $p^{\prime}: N \times N^{\prime} \rightarrow N^{\prime}$ are continuous, hence measurable. Thus the measurability of $g$ implies that $f=p \circ g$ and $f^{\prime}=p^{\prime} \circ g$ are measurable by Lemma 2.2. On the other hand, if $f$ and $f^{\prime}$ are measurable, then $g^{-1}\left(U \times U^{\prime}\right)=f^{-1}(U) \cap f^{\prime-1}\left(U^{\prime}\right)$ is a Borel set for all open sets $U \subset N$ and $U^{\prime} \subset N^{\prime}$. Since the products $U \times U^{\prime}$ form a base for open sets in $N \times N^{\prime}, g$ is measurable.

2.4. Lemma. Let $M$ be a manifold and $E$ a finite dimensional real vector space. If $f: M \rightarrow E$ and $g: M \rightarrow E$ are measurable maps, then $f+g$ : $M \rightarrow E$ is measurable, and $\lambda f: M \rightarrow E$ is measurable for each Barre function $\lambda$ in $M$.

Proof: The addition $E \times E \rightarrow E$ is continuous. Hence the measurability of $f+g$ follows from Lemmas 2.3 and 2.2. The proof is similar for $\lambda f$.

For the next lemma we recall that the set of linear maps $\mathbf{R}^{n} \rightarrow \mathbf{R}^{m}$ may be canonically represented by matrices of type $(m, n)$ with real entries. Hence to each map $M \rightarrow L\left(\mathbf{R}^{n}, \mathbf{R}^{m}\right)$ corresponds a matrix function $\left(\lambda_{i j}\right), \quad \lambda_{i j}: M \rightarrow \mathbf{R}, i=1, \ldots, m, j=1, \ldots, n$.

2.5. Lemma. Let $M$ be a manifold. Let $f: M \rightarrow L\left(\mathbf{R}^{n}, \mathbf{R}^{m}\right)$ be a map and $\left(\lambda_{i j}\right), i=1, \ldots, m, j=1, \ldots, n$, the corresponding matrix function. Then the following conditions are equivalent:

a) $f$ is measurable,

b) the functions $\lambda_{i j}$ are measurable, 
c) the map $g: M \times \mathbf{R}^{n} \rightarrow \mathbf{R}^{m}$ defined by

$$
g(x, v)=f(x)(v), \quad x \in M, v \in \mathbf{R}^{n},
$$

is measurable.

Proof: The evaluation map $e: L\left(\mathbf{R}^{n}, \mathbf{R}^{m}\right) \times \mathbf{R}^{n} \rightarrow \mathbf{R}^{m}$, defined by

$$
e(u, v)=u(v), \quad u \in L\left(\mathbf{R}^{n}, \mathbf{R}^{m}\right), \quad v \in \mathbf{R}^{n},
$$

is continuous. Hence a) implies c) by Lemmas 2.3 and 2.2.

Let $e_{j} \in \mathbf{R}^{n}, j=1, \ldots, n$, denote the $j$-th canonical basis vector $(0, \ldots, 1, \ldots, 0)$. If $\mathrm{c})$ holds, then the maps $f_{j}: M \rightarrow \mathbf{R}^{m}$, defined by $f_{j}(x)=g\left(x, e_{j}\right)$, are measurable. On the other hand $f_{j}=\lambda_{1 j} \times \ldots \times \lambda_{m j}$. Thus c) implies b).

Finally, b) implies a), since $f$ may be regarded as the cartesian product of the maps $\lambda_{i j}$.

2.6. Definition. Let $M$ be a manifold. A set $E \subset M$ is a null set, if $\varphi(E \cap U)$ has Lebesgue measure zero for each coordinate neighbourhood $U \subset M$ and each coordinate map $\varphi: U \rightarrow \mathbf{R}^{n}$ (of class $C^{\infty}$ ).

A condition is said to hold for almost every $x \in M$, or almost everywhere in $M$, if it holds everywhere except in a null set.

2.7. Remark. Since sets of Lebesgue measure zero in $\mathbf{R}^{n}$ are preserved by $C^{1}$-diffeomorphisms, the coordinate maps $\varphi$ may be of class $C^{1}$. In addition, only coordinate neighbourhoods $U$ belonging to a fixed cover $\left(U_{i}\right)$ of $M$ need be taken into consideration. We also remark that since $\left(U_{i}\right)$ may be chosen to be countable (Theorem 1.1. e)), the definition will agree with the measure defined later in section 3 .

2.8. Convention. As derivatives of functions differentiable almost everywhere we shall meet functions which are not defined in a Borel null set. If such a function is measurable in its set of definition its extension by a constant value will also be measurable. We agree to carry out always such an extension by the value 0 . Hence we may regard all functions as defined everywhere.

2.9. Lemma. Let $M$ and $N$ be Riemannian manifolds, $f: M \rightarrow N$ a continuous map, and $u: T M \rightarrow T N$ a measurable fibre map over $f$ (i.e. $u$ maps each fibre $T_{x} M, x \in M$, linearly into the fibre $\left.T_{f(x)} N\right)$. Then $\|u\|$ and $\operatorname{det} u$ are Baire functions in $M$.

Proof: In case $M=U$ and $N=V$ are open sets in $\mathbf{R}^{n}$ and $\mathbf{R}^{m}$ we may represent $u$ by a measurable map $g: U \rightarrow L\left(\mathbf{R}^{n}, \mathbf{R}^{m}\right)$ (Lemmas 2.3 and 2.5). The norm and the determinant are continuous functions in $L\left(\mathbf{R}^{n}, \mathbf{R}^{m}\right)$ which depend continuously on parameter points $x \in U$ and $y=f(x) \in V$. Hence the assertion follows by Lemma 2.2. Since the question is local, this also proves the general case. 
2.10. Theorem. Let $M$ and $N$ be manifolds and $f: M \rightarrow N$ a continuous map which is differentiable almost everywhere. Then the derivative map Df: $T M \rightarrow T N$ is measurable. If $M$ and $N$ are Riemannian manifolds, then $\|D f\|$ and $\operatorname{det} D f$ are Baire functions.

Proof: Since the question is local, we may assume that $M=U$ is an open subset of $\mathbf{R}^{n}$ and $f$ is a map of $U$ into $\mathbf{R}^{m}$. It is well-known that the partial derivatives of $f$ are measurable (cf. Saks [1], p. 170). Hence Df: $\quad U \times \mathbf{R}^{n} \rightarrow \mathbf{R}^{m} \times \mathbf{R}^{m}$ is measurable by Lemmas 2.5 and 2.3. The last assertion follows from Lemma 2.9 .

\section{Integration}

In this section we define a class of measures for Riemannian manifolds. Thereafter functions in manifolds may be integrated in accordance with standard methods (e.g. Munroe [1], Saks [1]). In particular, we follow Saks in defining integrals with infinite value.

3.1. Definition. Let $M$ be a manifold (cf. 1.2). A decomposition of $M$ is a pair $\left(\left(U_{i}\right),\left(E_{i}\right)\right)$, where $\left(U_{i}\right), i \in I$, is a cover of $M$ by coordinate neighbourhoods of class $C^{1}$ and $\left(E_{i}\right), i \in I$, is a family of Borel subsets of $M$. In addition, the following conditions are imposed:

a) the index set $I$ is countable,

b) for each $i \in I, E_{i} \subset U_{i}$,

c) $\cup E_{i}=M$ and $E_{i} \cap E_{j}=\varnothing$, for $i \neq j$.

A decomposition $\left(\left(V_{j}\right),\left(F_{j}\right)\right), j \in J$, is a refinement of a decomposition $\left(\left(U_{i}\right),\left(E_{i}\right)\right), i \in I$, if for each $j \in J$ there is an $i \in I$ such that $V_{j} \subset U_{i}$ and $F_{j} \subset E_{i}$.

3.2. Lemma. Every manifold $M$ has a decomposition and any two decompositions have a common refinement.

Proof: Since $M$ has a countable base for open sets, there is a cover $\left(U_{n}\right), n \in N$, of $M$ by coordinate neighbourhoods, indexed by the set of natural numbers. If we define

$$
E_{n}=U_{n}-\bigcup_{m=1}^{n-1} U_{m},
$$

then $E_{n}$ is a Borel set, and $\left(\left(U_{n}\right),\left(E_{n}\right)\right), n \in N$, a decomposition of $M$.

For the second assertion we remark that if $\left(\left(U_{i}\right),\left(E_{i}\right)\right), i \in I$, and $\left(\left(V_{j}\right),\left(F_{j}\right)\right), \quad j \in J$, are decompositions of $M$, then $\left(\left(U_{i} \cap V_{j}\right)\right.$, $\left.\left(E_{i} \cap F_{j}\right)\right), \quad(i, j) \in I \times J$, is also a decomposition.

3.3. Definition. Let $f: M \rightarrow N$ be a $C^{1}$-diffeomorphism of Riemannian manifolds $M$ and $N$. The continuous function $J_{f}=J(f)$ defined by 


$$
J_{f}(x)=|\operatorname{det} D f(x)|,
$$

is called the jacobian of $f$. It has the following properties:

a) if $f$ is the identity map, then $J_{f}=1$,

b) if $f: M \rightarrow N$ and $g: N \rightarrow L$ are $C^{1}$-diffeomorphisms of Riemannian manifolds $M, N$ and $L$, then

$$
J_{g \circ f}=\left(J_{g} \circ f\right) J_{f},
$$

c) if $f: M \rightarrow M^{\prime}$ and $g: N \rightarrow N^{\prime}$ are $C^{1}$-diffeomorphisms of Riemannian manifolds $M, M^{\prime}, N$ and $N^{\prime}$, then

$$
J_{f \times g}(x, y)=J_{f}(x) \cdot J_{g}(y), \quad x \in M, y \in N,
$$

for the map $f \times g: M \times N \rightarrow M^{\prime} \times N^{\prime}$.

3.4. Lemma. Let $\mu$ and $\tau$ be Borel measures in a manifold $M$. Let $\varphi$ be a non-negative Baire function in $M$, such that

$$
\mu(E)=\int_{E} \varphi d \tau
$$

for each Borel set $E \subset M$. Then a Baire function $\varrho$ in $M$ is $\mu$-integrable if and only if $\varrho \varphi$ is $\tau$-integrable and

$$
\int_{M} \varrho d \mu=\int_{M} \varrho \varphi d \tau .
$$

Proof: If $\varrho$ is a simple function, i.e., a finite linear combination of characteristic functions of Borel sets, then the proof is immediate. To prove the general case we may assume that $\varrho$ is non-negative. Then it is the limit of an increasing sequence of simple functions, and the assertion results from the monotone convergence theorem.

3.5. Theorem. With each Riemannian manifold $M$ we can associate a unique Borel measure $\tau_{M}$ so that the following conditions are satisfied:

a) if $N$ is an open Riemannian submanifold of a Riemannian manifold $M$, then $\tau_{M}(E)=\tau_{N}(E)$ for all Borel sets $E \subset N$,

b) if $f: M \rightarrow N$ is a $C^{1}$-diffeomorphism of Riemannian manifolds $M$ and $N$, then

$$
\tau_{N}(f(E))=\int_{E} J_{f} d \tau_{M}
$$

for all Borel sets $E \subset M$,

c) if $M=\mathbf{R}^{n}$ or $\mathbf{R}_{+}^{n}, n=1,2, \ldots, \tau_{M}$ is the Lebesgue measure.

Proof: Let $U$ and $V$ be open sets in $\mathbf{R}_{n}$ and $f: U \rightarrow V$ a $C^{1}$-diffeomorphism. Then it is well known that for all Borel sets $E \subset U$ 


$$
\tau(f(E))=\int_{E} J_{f} d \tau,
$$

where $\tau$ is the Lebesgue measure.

Let $M$ be a Riemannian manifold, such that there is a $C^{\mathbf{1} \text {-diffeo- }}$ morphism $f: U \rightarrow M$, where $U$ is an open set in $\mathbf{R}^{n}$. We define a measure $\tau_{f}$ in $M$ by

$$
\tau_{f}(E)=\tau\left(f^{-1}(E)\right)
$$

for all Borel sets $E \subset M$. If there are measures $\tau_{M}$ satisfying the conditions a)-c), then

$$
\tau_{M}(E)=\int_{E} J_{f} \circ f^{-1} d \tau_{f}
$$

must hold for all Borel sets $E \subset M$. On the other hand, the right hand side of (3.2) may be used for defining $\tau_{M}$, since it does not depend on the choice of $f$ and $U$ by (3.1) and Lemma 3.4. This definition is clearly compatible with a), and also with b) by Lemma 3.4 again.

Let $M$ be any Riemannian manifold and $\left(\left(U_{i}\right),\left(E_{i}\right)\right), i \in I$, a decomposition of $M$. If $\tau_{M}$ exists, it must satisfy

$$
\tau_{M}(E)=\sum_{I} \tau_{U_{i}}\left(E \cap E_{i}\right)
$$

for all Borel sets $E \subset M$, by a). In order to use (3.3) as a definition for $\tau_{M}$, we shall show that its right hand side does not depend on the choice of the decomposition $\left(\left(U_{i}\right),\left(E_{i}\right)\right)$. By Lemma 3.2 it is sufficient to consider a refinement $\left(\left(V_{j}\right),\left(F_{j}\right)\right), j \in J$, of $\left(\left(U_{i}\right),\left(E_{i}\right)\right), i \in I$.

Let $\alpha: J \rightarrow I$ be a map such that

$$
V_{j} \subset U_{\alpha(j)} \text { and } F_{j} \subset E_{\alpha(j)}
$$

for each $j \in J$. Let $J_{i}=\alpha^{-1}(i)$, for $i \in I$. Then we obtain

$$
E_{i}=\bigcup_{J_{i}} F_{j}
$$

and

$$
\sum_{J} \tau_{V_{j}}\left(E \cap F_{j}\right)=\sum_{I} \sum_{J_{i}} \tau_{U_{i}}\left(E \cap F_{j}\right)=\sum_{I} \tau_{U_{i}}\left(E \cap E_{i}\right)
$$

by a). Hence (3.3) may be used for defining $\tau_{M}$. The demonstration of the properties a) and b) results immediately from the following remarks:

1) If $\left(\left(U_{i}\right),\left(E_{i}\right)\right), i \in I$, is a decomposition of a manifold $M$ and $N$ is an open submanifold of $M$, then $\left(\left(U_{i} \cap N\right),\left(E_{i} \cap N\right)\right), i \in I$, is a decomposition of $N$. 
2) If $f: M \rightarrow N$ is a $C^{1}$-diffeomorphism of manifolds $M$ and $N$, and $\left(\left(U_{i}\right),\left(E_{i}\right)\right), i \in I$, a decomposition of $M$, then $\left(\left(f\left(U_{i}\right)\right),\left(f\left(E_{i}\right)\right)\right)$, $i \in I$, is a decomposition of $N$.

This completes the proof.

3.6. Definition. Let $M$ be a Riemannian manifold. The measure $\tau_{M}$ defined in Theorem 3.5 is called the Lebesgue measure of $M$.

3.7. Theorem. Let $M$ and $N$ be Riemannian manifolds. Then the Lebesgue measure of the Riemannian product manifold $M \times N$ is the product of the Lebesgue measures of $M$ and $N$.

Proof: By the definition of product measure, we must prove that

$$
\tau_{M \times N}(E \times F)=\tau_{M}(E) \tau_{N}(F)
$$

for all Borel sets $E \subset M$ and $F \subset N$.

If $M$ and $N$ are $\mathrm{C}^{\mathbf{1}}$-diffeomorphic to open subsets of $\mathbf{R}^{m}$ and $\mathbf{R}^{n}$, then $M \times N$ is $C^{1}$-diffeomorphic to an open subset of $\mathbf{R}^{m+n}$. In this case (3.4) follows from the definition (3.2) by Fubini's theorem and c) in Definition 3.2. In the general case it suffices to remark that if $\left(\left(U_{i}\right),\left(E_{i}\right)\right), i \in I$, and $\left(\left(V_{j}\right),\left(F_{j}\right)\right), j \in J$, are decompositions of $M$ and $N$, then $\left(\left(U_{i} \times V_{j}\right),\left(E_{i} \times F_{j}\right)\right),(i, j) \in I \times J$, is a decomposition of $M \times N$.

3.8. Theorem. Let $f: M \rightarrow N$ be a $C^{1}$-diffeomorphism of Riemannian manifolds $M$ and $N$. Then a Baire function $\varrho$ in $N$ is $\tau_{N^{-}}$integrable if and only if $(\varrho \circ f) J_{f}$ is $\tau_{M}$-integrable and

$$
\int_{N} \varrho d \tau_{N}=\int_{M}(\varrho \circ f) J_{f} d \tau_{M} .
$$

Proof: This follows immediately from Lemma 3.4 and b) of Theorem 3.5.

\section{Curves and are length}

4.1. Definition. A curve in a manifold $M$ is a continuous map $\gamma$ from a closed interval $I=[a, b], a \leq b$, to $M$. A curve $\gamma$ is called differentiable if $\gamma$ is continuously differentiable.

4.2. Remark. If $\gamma: I \rightarrow M$ is a differentiable curve, we identify the derivative $D \gamma(t), t \in I$, with a tangent vector of $M$ at $\gamma(t)$. This determines a curve $D \gamma$ in the tangent bundle $T M$.

4.3. Definition. Let $M$ be a Riemannian manifold and $\gamma$ a piecewise differentiable curve in $M$. The length of $\gamma$ is defined by

$$
l(\gamma)=\int_{I}\|D \gamma(t)\| d t,
$$

where || || refers to the Riemannian metric . 
4.4. Definition. Let $M$ be a Riemannian manifold and $x, y$ two points of $M$. Their distance $d(x, y)$ is defined by

$$
d(x, y)=\inf _{\Gamma} l(\gamma)
$$

where $\Gamma$ is the family of piecewise differentiable curves $\gamma:[a, b] \rightarrow M$ joining $x$ and $y$, i.e. $\gamma(a)=x, \gamma(b)=y$. Since $M$ is connected, $\Gamma$ is not empty.

4.5. Lemma. The distance $d(x, y)$ is a metric in $M$ compatible with its topology.

The proof is elementary. We remark that this proves the metrizability of $M$ independently of Urysohn's theorem.

4.6. Lemma. Let $\gamma:[a, b] \rightarrow M$ be a curve in a Riemannian manifold $M$. If $\gamma$ is differentiable at $t_{0} \in[a, b]$, then the interval function

$$
\left[t, t^{\prime}\right] \rightarrow d\left(\gamma(t), \gamma\left(t^{\prime}\right)\right), \quad a \leq t \leq t^{\prime} \leq b,
$$

is differentiable at $t_{0}$ and its derivative is $\left\|D \gamma\left(t_{0}\right)\right\|$. In particular, if $\gamma$ is differentiable,

$$
l(\gamma)=\sup \sum d\left(\gamma\left(t_{i}\right), \gamma\left(t_{i+1}\right)\right), \quad a=t_{0} \leq t_{1} \leq \ldots \leq t_{n}=b .
$$

Proof: If $M$ is locally cuclidean, the proof is well-known. In the general case we may replace the Riemannian metric $g$ of $M$ by a euclidean metric coinciding with $g$ at $\gamma\left(t_{0}\right)$. If $\gamma$ is differentiable, we denote by $s(t)$ the right hand side of (4.1) for the restriction of $\gamma$ to $[a, t]$, when $a \leq t \leq b$. Then $s$ is continuously differentiable and its derivative is $\|D \gamma(t)\|$. Hence $s(b)=l(\gamma)$.

4.7. Definition. Let $\gamma:[a, b] \rightarrow M$ be a curve in a Riemannian manifold $M$. The length of $\gamma$ is defined by

$$
l(\gamma)=\sup \sum d\left(\gamma\left(t_{i}\right), \gamma\left(t_{i+1}\right)\right), \quad a=t_{0} \leq t_{1} \leq \ldots \leq t_{n}=b .
$$

If $l(\gamma)$ is finite, $\gamma$ is rectifiable.

Let $\gamma$ be rectifiable and let $s(t)$ denote the length of the restriction of $\gamma$ to $[a, t], a \leq t \leq b$. The function $s$ is called the arc length of $\gamma$. There is a unique curve $\gamma_{1}:[0, l(\gamma)] \rightarrow M$ with the property $\gamma=\gamma_{1} \circ s$. The curve $\gamma_{1}$ is called the parametrization of $\gamma$ by arc length.

4.8. Lemma. Let $\gamma$ be a rectifiable curve in a Riemannian manifold $M$. Then $\gamma$ is differentiable almost everywhere.

Proof: If $f$ is a lipschitzian function in $M$, then $f \circ \gamma$ is of bounded variation, and hence differentiable almost everywhere. Since local coordinates in $M$ are lipschitzian, at least locally, the assertion follows.

4.9. Theorem. Let $\gamma$ be a rectifiable curve in a Riemannian manifold $M$ and let $s$ denote its arc length. Then the equality 


$$
s^{\prime}(t)=\|D \gamma(t)\|
$$

holds almost everywhere. In particular, for the parametrization $\gamma_{1}$ of $\gamma$ by arc length, we have

$$
\left\|D \gamma_{1}(s)\right\|=1 \text { a.e. }
$$

Proof: This follows from Lemmas 4.6 and 4.8 (Riesz--Sz.-Nagy [1], pp. 23, 25).

4.10. Definition. Let $\gamma$ be a rectifiable curve and $气$ a Baire function in a Riemannian manifold $M$. Let $\gamma_{1}$ be the parametrization of $\gamma$ by arc length. The integral of $\varrho$ along $\gamma$ is defined by

$$
\int_{\gamma} \varrho d s=\int_{0}^{l(\gamma)} \varrho \circ \gamma_{1} d s,
$$

provided the latter integral exists. Otherwise the integral of $\varrho$ along $\gamma$ is undefined.

4.11. Theorem. Let $M$ and $N$ be Riemannian manifolds and $f$ : $M \rightarrow N$ a continuously differentiable map. Let $\gamma$ be a rectifiable curve in $M$. Then $\gamma^{\prime}=f \circ \gamma$ is rectifiable and for each non-negative Baire function $\varrho$ in $N$

$$
\int_{\gamma^{\prime}} \varrho d s \leq \int_{\gamma}(\varrho \circ f)\|D f\| d s .
$$

Proof: Since $f$ is locally lipschitzian, there is a constant $C$ such that

$$
l\left(\gamma^{\prime}\right) \leq C l(\gamma)
$$

and the same relation holds for subcurves of $\gamma$ and their images under $f$. Let $s\left(s^{\prime}\right)$ be the arc length and $\gamma_{1}\left(\gamma_{1}^{\prime}\right)$ the parametrization by arc length of $\gamma\left(\gamma^{\prime}\right)$. Then there is a unique monotone function $\varphi:[0, l(\gamma)] \rightarrow$ $\left[0, l\left(\gamma^{\prime}\right)\right]$ with the property $s^{\prime}=\varphi \circ s$. By (4.2) $\varphi$ is absolutely continuous. Hence we obtain

$$
\int_{\gamma^{\prime}} \varrho d s=\int_{0}^{l\left(\gamma^{\prime}\right)} \varrho \circ \gamma_{1}^{\prime} d s^{\prime}=\int_{0}^{l(\gamma)}\left(\varrho \circ \gamma_{1}^{\prime} \circ \varphi\right) \varphi^{\prime} d s .
$$

But $\gamma_{1}^{\prime} \circ \varphi=f \circ \gamma_{1}$ and $\varphi^{\prime}==\left\|D \gamma_{1}^{\prime}\right\| \varphi^{\prime}=\left\|D f \circ D \gamma_{1}\right\| \leq\|D f\|$ a.e. This proves the theorem. 


\section{Module}

In this section we define the module of a family of curves in a Riemannian manifold and study its properties.

5.1. Definition. Let $M$ be a Riemannian manifold and $\Gamma$ a family of curves in $M$. We denote by $F(\Gamma)$ the class of all non-negative Baire functions $\varrho$ in $M$ which satisfy

$$
\int_{\gamma} \varrho d s \geq 1
$$

for all rectifiable curves $\gamma \in \Gamma$.

For each positive real number $p$ we define the $p$-module of $\Gamma$ as follows

$$
M_{p}(\Gamma)=\inf _{F(\Gamma)} \int_{M} \varrho^{p} d \tau,
$$

where $\tau$ denotes the Lebesgue measure of $M$. If $F(\Gamma)$ is empty, we define $M_{p}(\Gamma)=\infty$.

5.2. Lemma. The module $M_{p}$ is monotone and countably subadditive, i.e.

$$
M_{p}(\Gamma) \leq M_{p}\left(\Gamma^{\prime}\right)
$$

if $\Gamma \subset \Gamma^{\prime}$, and

if $\Gamma=\bigcup_{i} \Gamma_{i}, \quad i=1,2, \ldots$

$$
M_{p}(\Gamma) \leq \sum_{i} M_{p}\left(\Gamma_{i}\right)
$$

Proof. If $\Gamma \subset \Gamma^{\prime}$, then clearly $F(\Gamma) \supset F\left(\Gamma^{\prime}\right)$. This proves the inequality (5.1.).

To prove (5.2) we choose functions $\varrho_{i} \in F\left(\Gamma_{i}\right), i=1,2, \ldots$ Then, for each $n \geq 1, \quad \sigma_{n}=\sup \left\{\varrho_{1}, \ldots, \varrho_{n}\right\} \in F\left(\Gamma_{1} \cup \ldots \cup \Gamma_{n}\right)$ and

$$
\int_{M} \sigma_{n}^{p} d \tau \leq \sum_{i=1}^{n} \int_{M} \iota_{i}^{p} d \tau .
$$

Choose $\varepsilon>0$. If $\sum M_{p}\left(\Gamma_{i}\right)$ is finite, we may assume that

$$
\sum_{i} \int_{M} \varrho_{i}^{p} d \tau<\sum_{i} M_{p}\left(\Gamma_{i}\right)+\varepsilon .
$$

Then $\varrho=\lim \sigma_{i}$ belongs to $F(\Gamma)$ and

$$
\int_{M} \epsilon^{p} d \tau \leq \sum_{i} M_{p}\left(\Gamma_{i}\right)+\varepsilon
$$

by the monotone convergence theorem. This completes the proof. 
5.3. Theorem. Let $f: N \rightarrow N^{\prime}$ be a $C^{1}$-diffeomorphism of manifolds $N$ and $N^{\prime}$ with Riemannian metrics $g$ and $g^{\prime}$ satisfying

$$
m^{2} g \leq f^{*} g^{\prime} \leq M^{2} g, \quad 0<m \leq M<\infty .
$$

Let $\Gamma$ be a family of curves in $N$ and let $\Gamma^{\prime}$ denote its image under $f$ in $N^{\prime}$. Then

$$
\frac{m^{n}}{M^{p}} M_{p}(\Gamma) \leq M_{p}\left(\Gamma^{\prime}\right) \leq \frac{M^{n}}{m^{p}} M_{p}(\Gamma),
$$

where $n$ is the dimension of $N$. In addition, if $f$ is conformal,

$$
M^{n-p} M_{p}(\Gamma) \leq M_{p}\left(\Gamma^{\prime}\right) \leq m^{n-p} M_{p}(\Gamma), \quad \text { for } p \geq n,
$$

and

$$
m^{n-p} M_{p}(\Gamma) \leq M_{p}\left(\Gamma^{\prime}\right) \leq M^{n-p} M_{p}(\Gamma), \quad \text { for } p \leq n .
$$

Proof: Let $\varrho \in F\left(\Gamma^{\prime}\right)$. Let $\gamma \in \Gamma$ be a rectifiable curve. Then, by Theorem 4.11,

$$
1 \leq \int_{\gamma}(\varrho \circ f)\|D f\| d s .
$$

Hence $(\varrho \circ f)\|D f\| \in F(T)$.

Let $\tau$ and $\tau^{\prime}$ denote the Lebesgue measures of $N$ and $N^{\prime}$, respectively. Then

$$
\int_{N^{\prime}} \varrho^{p} d \tau^{\prime}=\int_{N}(\varrho \circ f)^{p} J_{f} d \tau
$$

by Theorem $3.5 \mathrm{~b}$ ) and Lemma 3.4. The assumption (5.3) implies $J_{f} \geq m^{n}$ and $\|D f\| \leq M$. Hence

$$
\frac{m^{n}}{M^{p}} \int_{N}(\varrho \circ f)^{p}\|D f\|^{p} d \tau \leq \int_{N^{\prime}} \varrho^{p} d \tau^{\prime} .
$$

This proves the first inequality of (5.4). The second inequality follows by interchanging $N$ and $N^{\prime}$.

If $f$ is conformal, we have $J_{f}=\|D f\|^{n}$. Then

$$
\|D f\|^{n-p} \int_{N}(\varrho \circ f)^{p}\|D f\|^{p} d \tau=\int_{N^{\prime}} \varrho^{p} d \tau^{\prime} .
$$

Hence the left hand inequalities in (5.5) and (5.6) result from the estimate $m \leq\|D f\| \leq M$. The remaining inequalities are again proved by interchanging $N$ and $N^{\prime}$. 
5.4. Notation. Let $S=S^{n-1}$ be the unit sphere in the euclidean space $\mathbf{R}^{n}$. We denote by $S_{+}=S_{+}^{n-1}$ the hemisphere $\left\{x \in S \mid x_{1} \geq 0\right\}$ and by $\Gamma_{+}$the family of curves joining the points $a=(0, \ldots, 0,1)$ and $b=(0, \ldots, 0,-1)$ in $S_{+}$.

We denote by $S_{+}^{\prime}$ and $(n-1)$-dimensional spherical cap contained in a hemisphere, or, as a limiting case, an $(n-1)$-dimensional disc. We denote by $\Gamma^{+}$the family of curves in $S_{+}^{\prime}$ which join a pair of opposite boundary points.

5.5. Lemma. There is a conformal diffeomorphism $f: S_{+} \rightarrow S_{+}^{\prime}$ with the property $f\left(\Gamma_{+}\right)=\Gamma_{+}^{\prime}$.

Proof: We may assume without loss of generality that the boundary spheres of $S_{+}$and $S_{+}^{\prime}$ coincide, since this may be achieved by a conformal transformation. Then $f$ is obtained by projecting from the point which divides the segment joining the centers of $S_{+}$and $S_{+}^{\prime}$ in the ratio of the respective radii.

5.6. Theorem. The $n$-modules of the families $\Gamma_{+}$and $\Gamma_{+}^{\prime}$ defined in 5.4 satisfy the inequalities

$$
M_{n}\left(\Gamma_{+}\right) \geq \frac{\omega_{n-1}}{2[(n-1) \pi]^{n-1}}=\left[\Gamma\left(\frac{n-1}{2}\right) \pi^{\frac{n-1}{2}}(n-1)^{n-1}\right]^{-1}
$$

and

$$
M_{n}\left(\Gamma_{+}^{\prime}\right) \geq \frac{2 M_{n}\left(\Gamma_{+}\right)}{d},
$$

where $d$ is the diameter of $S_{+}^{\prime}$.

Proof: For each point $y \in S_{+}^{n-2}$ we define the curve $\gamma_{y}:[0, \pi] \rightarrow S_{+}$by

$$
\gamma_{y}(\varphi)=\cos \varphi \cdot a+\sin \varphi \cdot y, \quad 0 \leq \varphi \leq \pi .
$$

Clearly, $\gamma_{y}$ is parametrized by arc length.

Let $\varrho \in F\left(\Gamma_{+}\right)$. Since $\gamma_{y} \in \Gamma_{+}$, we have

$$
\int_{\gamma_{y}} \varrho d \varphi \geq 1
$$

for each point $y \in S_{+}^{n-2}$. By Hölder's inequality we obtain

$$
\int_{\gamma_{y}} \varrho^{n}(\sin \varphi)^{n-2} d \varphi\left(\int_{0}^{\pi}(\sin \varphi)^{-\frac{n-2}{n-1}} d \varphi\right)^{n-1} \geq 1 .
$$

Since $\sin \varphi \geq \frac{2}{\pi} \varphi$ for $0 \leq \varphi \leq \frac{\pi}{2}$, the last integral can be estimated: 


$$
2 \int_{0}^{\frac{\pi}{2}}(\sin \varphi)^{-\frac{n-2}{n-1}} d \varphi \leq 2\left(\frac{\pi}{2}\right)^{\frac{n-2}{n-1}} \int_{0}^{\frac{\pi}{2}} \varphi^{-\frac{n-2}{n-1}} d \varphi=(n-1) \pi .
$$

Hence, for $y \in S_{+}^{n-2}$,

$$
\int_{\gamma_{y}} \varrho^{n} \sin ^{n-2} \varphi d \varphi \geq \frac{1}{[(n-1) \pi]^{n-1}}
$$

and

$$
\int_{s_{+}} \varrho^{n} d \tau \geq \frac{\omega_{n-1}}{2[(n-1) \pi]^{n-1}} .
$$

This proves the inequality (5.7).

To prove (5.8) we note that the conformal diffeomorphism $f$ given in Lemma 5.5. satisfies the conditions of Theorem 5.3 with $M=d / 2, m=d / 4$.

5.7. Remark. The exact value of the last integral in (5.9) is given by

$$
\sqrt{\pi} \cdot \Gamma\left(\frac{1}{2(n-1)}\right)\left[\Gamma\left(\frac{1}{2}+\frac{1}{2(n-1)}\right)\right]^{-1} .
$$

This is asymptotically equal to $2(n-1)$ for large values of $n$.

5.8. Corollary. Let $F_{1}$ and $F_{2}$ be disjoint non-empty subsets of an (n-1)-sphere $S$ of radius $R$. Let $\Gamma$ be the family of curves which join $F_{1}$ and $F_{2}$ in $S$. Then

$$
M_{n}(\Gamma) \geq \frac{k}{R}
$$

where

$$
\frac{1}{k}=[(n-1) \sqrt{\pi}]^{n-1} \Gamma\left(\frac{n-1}{2}\right) .
$$

Proof: Let $a \in F_{1}$ and $b \in F_{2}$. Let $S^{\prime} \subset S$ be the cap of height $\leq R$ with $a$ and $b$ as opposite boundary points. Then $\Gamma$ contains the family $\Gamma^{\prime}$ of curves joining $a$ and $b$ in $S^{\prime}$. Hence it is sufficient to prove $(5.10)$ for $\Gamma^{\prime}$. But this follows immediately from (5.8) and the inequality $d \leq 2 R$.

5.9. Theorem. Let $a$ and $b$ be two points of the sphere $S^{n-1}$. Let $C_{1}$ and $C_{2}$ be disjoint connected subsets of $\mathbf{R}^{n}$, such that $C_{1}$ contains $a$ and the origin and $C_{2}$ is unbounded and contains $b$. Let $\Gamma$ be the family of curves joining $C_{1}$ and $C_{2}$ in $\mathbf{R}^{n}$. Then

$$
M_{n}(\Gamma) \geq \varkappa
$$

where $\varkappa=\varkappa_{n}$ depends only on $n$. 
Proof: Let us first suppose that $|a-b| \geq 1$. Let $S_{r}$ denote the sphere $\left\{x|| x-\frac{1}{2} b \mid=r\right\}$ and let $\Gamma_{r}$ be the family of curves which join $C_{1}$ and $C_{2}$ in $S_{r}$. Then $\Gamma_{r} \subset \Gamma$ and if $\varrho \in F(\Gamma)$, then $\varrho \mid S_{r} \in F\left(\Gamma_{r}\right)$, for $\frac{1}{2}<r<\frac{1}{2} \sqrt{3}$. Hence, by Corollary 5.8,

$$
\int_{S_{r}} \varrho^{n} d \sigma \geq \frac{k}{r},
$$

where $\sigma$ denotes the Lebesgue measure of $S_{r}$. Integrating with respect to $r$ we obtain

$$
\int_{\mathbf{R}^{n}} \varrho^{n} d \tau \geq \int_{\frac{1}{2}}^{\frac{\sqrt{3}}{2}} \frac{k}{r} d r=\frac{k}{2} \log 3 .
$$

If $|a-b|<1$, the same inequality results by considering the spheres $\left\{x|| x-\frac{1}{2}(a+b) \mid=r\right\}, \frac{1}{2}<r<\frac{1}{2} \sqrt{3}$.

This proof is taken from Väisälä [1].

5.10. Theorem. Let $\Gamma$ be a family of curves in $\mathbf{R}^{n}$ with the following property: For each $\gamma: I \rightarrow \mathbf{R}^{n}$ in $\Gamma$ there are points $a, b \in I$ such that $|\gamma(a)|=m$ and $|\gamma(b)|=M, 0<m \leq M$. Then

$$
M_{n}(\Gamma) \leq \omega_{n}\left(\log \frac{M}{m}\right)^{1-n}
$$

Equality holds when $\Gamma$ contains the curves $\gamma_{x}, x \in S^{n-1}$, defined by

$$
\gamma_{x}(r)=r x
$$$$
m \leq r \leq M \text {. }
$$

Proof: We define the function $\varrho$ by

$$
\varrho(x)= \begin{cases}\left(|x| \cdot \log \frac{M}{m}\right)^{-1}, & \text { for } m \leq|x| \leq M, \\ 0 & \text { otherwise } .\end{cases}
$$

Then $\varrho \in F(\Gamma)$ and

$$
\int_{\mathbf{R}^{n}} \varrho^{n} d \tau=\omega_{n}\left(\log \frac{M}{m}\right)^{1-n}
$$

which proves (5.12).

Let us now assume that $\Gamma$ contains the curves $\gamma_{x}$. Let $\varrho \in F(\Gamma)$. Then 


$$
1 \leq \int_{\gamma_{x}} \varrho d s=\int_{m}^{M} \varrho(r x) d r
$$

for each $x \in S^{n-1}$. Thus

$$
1 \leq \int_{m}^{M} \varrho^{n} r^{n-1} d r\left(\int_{m}^{M} \frac{1}{r} d r\right)^{n-1}
$$

by Hölder's inequality. Hence

$$
\int_{\mathbf{R}^{n}} \varrho^{n} d \tau \geq \omega_{n}\left(\log \frac{M}{m}\right)^{1-n}
$$

which completes the proof.

\section{6. $A C L^{p}$-functions}

6.1. Definition. Let $M$ be a Riemannian manifold. A differential $u$ in $M$ is a Baire function $u: T M \rightarrow \mathbf{R}$ which is linear in each fibre $T_{x} M$, $x \in M$. A differential $u$ in $M$ is an $L^{p}$-differential, with $p \geq 1$, if the Baire function $\|u\|^{p}$ (cf. Lemma 2.9) is integrable over any compact set $A \subset M$.

6.2. Remark. The concept of an $L^{p}$-differential is independent of the Riemannian metric chosen for the definition. Hence $C^{1}$-diffeomorphisms of Riemannian manifolds transform $L^{p}$-differentials into $L^{p}$-differentials.

6.3. Definition. Let $M$ and $N$ be Riemannian manifolds. A continuous function $f: M \rightarrow \mathbf{R}$ is $A C L^{p}$, for some $p \geq 1$, if there is an $L^{p}$-differential $u$ in $M$ with the following property:

(6.1.) $f \circ \gamma_{1}$ is absolutely continuous and $D\left(f \circ \gamma_{1}\right)=u \circ D \gamma_{1}$ a.e.

for the parametrization by arc length $\gamma_{1}$ of all rectifiable curves $\gamma: I \rightarrow M$ except for a family $\Gamma$ with $M_{p}(\Gamma)=0$.

In this case, $u$ is called the differential of $f$ and denoted by $D f$. It is uniquely defined up to a set of measure zero.

A continuous map $f: M \rightarrow N$ is $A C L^{p}$, if $g \circ f$ is $A C L^{p}$ for each $C^{1}$-function $g$ in $N$.

6.4. Remark. Let $M, N$ and $L$ be Riemannian manifolds. If $f$ : $M \rightarrow N$ is an $A C L^{p}$-map and $g: N \rightarrow L$ a $C^{1}$-map, then $g \circ f$ is $A C L^{p}$. Similarly, it may be shown that if $f: M \rightarrow N$ is $A C L^{p}$ and $g: L \rightarrow M$ a $C^{1}$-diffeomorphism, then $f \circ g$ is $A C L^{p}$ (cf. Remark 6.2, Theorem 5.3). This also holds if $g: M \times L \rightarrow M$ is a projection. 
The classical definition of $A C L^{p}$-functions is applicable only for open subsets of $\mathbf{R}^{n}$, since it makes essential use of coordinates. In order to prove that our definition implies the classical definition, it suffices to apply (6.1) to segments parallel to the coordinate axes. For the inverse implication the partial derivatives of the function in question are combined to form an $L^{P}$-differential and the proof is completed by using Lemma 4.1 in Väisälä $[1]$.

6.5. Lemma. Let $M$ be a Riemannian manifold and $f: M \rightarrow \mathbf{R}^{n} \quad a$ map whose components $f_{i}: M \rightarrow \mathbf{R}, i=1, \ldots, n$, are $A C L^{p}$-functions. If $U \subset \mathbf{R}^{n}$ is an open set containing $f(M)$, and $g: U \rightarrow \mathbf{R}$ a $C^{\mathbf{1} \text {-function, }}$ then $g \circ f$ is an $A C L^{p}$-function in $M$.

Proof: Let $D_{i} g, i=1, \ldots, n$, denote the partial derivatives of $g$. Then

is an $L^{p}$-differential in $M$.

$$
u=\sum_{i=1}^{n}\left(D_{i} g \circ f\right) D f_{i}
$$

Let $\gamma$ be a rectifiable curve in $M$ such that the condition (6.1) is satisfied for each function $f_{i}$. Then it may be immediately verified that (6.1.) holds for $\gamma, g \circ f$ and $u$.

6.6. Remark. Lemma 6.5 shows that an $A C L^{p}$-function $f: M \rightarrow \mathbf{R}$ is also an $A C L^{p}$-map.

6.7. Theorem. Let $M$ and $N$ be Riemannian manifolds and $f: M \rightarrow N$ a map. If each point $x \in M$ has a neighbourhood $U$ such that $f \mid U$ : $U \rightarrow V$ is $A C L^{p}$ for some neighbourhood $V$ of $f(x)$ containing $f(U)$, then $f$ is $A C L^{p}$.

Proof: Let $g: N \rightarrow \mathbf{R}$ be a $C^{1}$-function. We are to show that $g \circ f$ is $A C L^{p}$.

Let $\left(U_{i}\right), i=1,2, \ldots$, be a cover of $M$ by open sets $U_{i}$ such that $f \mid U_{i}: U_{i} \rightarrow V_{i}$ is $A C L^{p}$ for some open set $V_{i} \subset N$. Then $g \circ f \mid U_{i}$ is $A C L^{p}$. Let $u_{i}$ denote its differential. Since $u_{i}=u_{j}$ a.e. in $U_{i} \cap U_{j}$, there is an $L^{p}$-differential $u$ in $M$ such that $u_{i}=u$ a.e. in $U_{i}$.

Let $\Gamma_{i}, i=1,2, \ldots$, denote the family of rectifiable curves $\gamma$ in $U_{i}$ such that (6.1) does not hold for $\gamma, g \circ f$ and $u$. Then $M_{p}\left(\Gamma_{i}\right)=0$ for $i=1,2, \ldots$, and $M_{p}(\Gamma)=0$ for $\Gamma=\cup \Gamma_{i}$. This completes the proof, since every rectifiable curve in $M$ for which (6.1) is not satisfied, has a subcurve in $\Gamma$.

6.8. Lemma. Let $M$ and $N$ be Riemannian manifolds and $f: M \rightarrow N$ an $A C L^{p}$-map. Then each point $y \in N$ has a coordinate neighbourhood $V$ such that, if $U=f^{-1}(V)$, the map $f \mid U: U \rightarrow V$ is $A C L^{p}$.

Proof: Let $W \subset N$ be a coordinate neighbourhood of $y$ with coordinate functions $y_{i}: W \rightarrow \mathbf{R}, i=1, \ldots, n$. Let $\varphi$ be a $C^{1}$-function 
in $N$, with supp $\varphi \subset W$, having value 1 in a neighbourhood $V$ of $y$. Then the functions $\varphi y_{i}, i=1, \ldots, n$, may be extended to $C^{1}$-functions $z_{i}: N \rightarrow \mathbf{R}$ such that $z_{i} \circ f$ is $A C L^{p}$.

Let $f^{-1}(V)$ be denoted by $U$. Then each function $y_{i} \circ f\left|U=z_{i} \circ f\right| U$ is $A C L^{p}$. Every $C^{1}$-function $g: V \rightarrow \mathbf{R}$ may be represented in the form $g=h\left(y_{1}, \ldots, y_{n}\right)$, where $h$ is a $C^{1}$-function. Hence $g \circ f \mid U$ is $A C L^{p}$ by Lemma 6.5 .

6.9. Theorem. Let $M, N$ and $L$ be Riemannian manifolds. Let $f$ : $M \times N \rightarrow L$ be an $A C L^{p}$-map. Then the map $f_{y}: M \rightarrow L, y \in N$, defined by $f_{y}(x)=f(x, y), x \in M$, is $A C L^{p}$ for almost every $y \in N$.

Proof: We assume first that $f$ is a function. Let $\Gamma$ denote the family of rectifiable curves in $M \times N$ for which (6.1) does not hold. Let $\Gamma_{y} \subset \Gamma$, $y \in \Lambda$, be the subfamily of curves contained in $M \times\{y\}$. It is sufficient to show that $M_{p}\left(\Gamma_{y}\right)=0$ in $M \times\{y\}$ for almost every $y \in N$.

We denote

$$
M(y)=\inf _{\varrho \in F(\Gamma)} \int_{M \times\{y\}} \varrho^{p} d \sigma,
$$

Then $M_{p}\left(\Gamma_{y}\right) \leq M(y)$. Since $M_{p}(\Gamma)=0$, the measure of the set $\{y \in N$ $M(y) \geq d\}$ must zero for each $d>0$. Hence $M(y)=0$ almost everywhere and the proof is complete in case $f$ is a function.

If $L$ is diffeomorphic to an open set in $\mathbf{R}^{n}$, the proof follows from the first part by Lemma 6.5.

In the general case we cover $L$ by coordinate neighbourhoods $W_{j}$, $j \in J$, such that $f \mid f^{-1}\left(W_{j}\right): f^{-1}\left(W_{j}\right) \rightarrow W_{j}$ is $A C L^{p}$ (Lemma 6.8). Let $\left(U_{i} \times V_{i}\right), i \in I$, be a countable refinement of $\left(f^{-1}\left(W_{j}\right)\right), j \in J$. Then, for each $i \in I, f_{y} \mid U_{i}$ is $A C L^{p}$ for almost every $y \in V_{i}$ by what we have proved above. This completes the proof by Theorem 6.7.

6.10. Theorem. Let $M$ be a Riemannian manifold and $f: M \rightarrow \mathbf{R}$ a continuous function which is $A C L^{p}$ in an open set $U \subset M$. Then there is a sequence $f_{i}, i=1,2, \ldots$, of $C^{\infty}$-functions in $M$ such that $f_{i} \rightarrow f$ uniformly in compact sets and, in addition, $D f_{i} \rightarrow D f$ in $L^{p}$ in each compact set $A \subset U$.

Proof: If $M=\mathbf{R}^{n}$, the proof is carried out in a well-known way by means of convolutions (cf. Lehto-Virtanen [1], p. 152). In the general case partitions of unity are used.

6.11. Remark. A similar theorem holds for $A C L^{p}$-maps, but we neither state nor prove it, since we can avoid it (cf. Theorem 6.13).

6.12. Lemma. Let $B=\left\{x \in \mathbf{R}^{n}|a<| x \mid<b\right\}$, for some $0<a<b$. Let $S_{r}=\left\{x \in \mathbf{R}^{n}|| x \mid=r\right\}$ for $r>0$ and $S_{1}=S$. Then, if $f: B \rightarrow S$ is a $C^{2}$-map, the integral 


$$
\int_{S_{r}} \operatorname{det} D\left(f \mid S_{r}\right) d \sigma
$$

is independent of $r, a<r<b$.

Proof: Let $e_{1}, \ldots, e_{n}$ be the standard orthonormal basis in $\mathbf{R}^{n}$. Let $\mathcal{\perp}:\left(\mathbf{R}^{n}\right)^{n} \rightarrow \mathbf{R}$ be an alternating multilinear function with $\Delta\left(e_{1}, \ldots, e_{n}\right)=1$. We define a $C^{\mathbf{1}}$-vector field $v(x)=\sum v^{i}(x) e_{i}$ in $B$ by

$$
v^{i}(x)=(-1)^{i-1} \Delta\left(f(x), f^{\prime}(x) e_{1}, \ldots, f^{\prime}(x) e_{i}, \ldots, f^{\prime}(x) e_{n}\right) .
$$

Then

$$
\begin{aligned}
I\left(v(x), e_{1}, \ldots, \hat{e}_{i}, \ldots, e_{n}\right) & =\Delta\left(v^{i}(x) e_{i}, e_{1}, \ldots, \hat{e}_{i}, \ldots, e_{n}\right) \\
& =\Delta\left(f(x), f^{\prime}(x) e_{1}, \ldots, f^{\prime}(x) e_{i}, \ldots, f^{\prime}(x) e_{n}\right)
\end{aligned}
$$

and

$$
\Delta\left(v(x), v_{1}, \ldots, v_{n-1}\right)=\Delta\left(f(x), f^{\prime}(x) v_{1}, \ldots, f^{\prime}(x) v_{n-1}\right)
$$

results by linearity for any vectors $v_{1}, \ldots, v_{n-1} \in \mathbf{R}^{n}$.

Let $x \in S_{r}$. Choosing for $v_{1}, \ldots, v_{n-1}$ in (6.2) an orthonormal set, orthogonal to $x$, it can be seen that $\operatorname{det} D\left(f \mid S_{r}\right)(x)$ is equal to the normal component of $v(x)$ to $S_{r}$. Hence the assertion follows if we show that $\operatorname{div} v=\sum D_{i} v^{i}=0$ in $B$.

We may write

$$
v^{i}(x)=\Delta\left(f^{\prime}(x) e_{1}, \ldots, f^{\prime}(x) e_{i-1}, f(x), f^{\prime}(x) e_{i+1}, \ldots, f^{\prime}(x) e_{n}\right) .
$$

Hence, by direct calculation,

and

$$
D_{i} v^{i}=\Delta\left(f^{\prime} e_{1}, \ldots, f^{\prime} e_{n}\right)+\sum_{j \neq i} \Delta\left(f^{\prime} e_{1}, \ldots, f^{\prime \prime}\left(e_{j}, e_{i}\right), \ldots, f, \ldots, f^{\prime} e_{n}\right)
$$

$$
\sum D_{i} v^{i}=n \Delta\left(f^{\prime} e_{1}, \ldots, f^{\prime} e_{n}\right)=0
$$

since $f^{\prime}(x)$ maps $\mathbf{R}^{n}$ into a space of dimension $n-1$.

6.13. Theorem. Let $f: S^{n} \rightarrow S^{n}$ be an $A C L^{n}$-map. If $f$ is homotopic to a diffeomorphism, then

$$
\int_{S^{n}} \operatorname{det} D f d \sigma= \pm \omega_{n+1} .
$$

Here $D f$ may be defined by components, since $S^{n} \subset \mathbf{R}^{n+1}$.

Proof: Let $A=\left\{x \in \mathbf{R}^{n+1}|1 \leq| x \mid \leq 2\right\}$. By hypothesis there is a continuous map $h: A \rightarrow S^{n}$ such that $h \mid S^{n}=f$ and the map $g: S^{n} \rightarrow S^{n}$, defined by $g(x)=h(2 x), \quad x \in S^{n}$, is a diffeomorphism. 
If $h$ is a $C^{2}$-map, then the assertion follows from Lemma 6.12 and Theorem 3.8. Otherwise, we first extend $h$ to $\mathbf{R}^{n+1}-\{0\}$ by

$$
h(x)= \begin{cases}f(x /|x|), & \text { if }|x|<1, \\ g(x /|x|), & \text { if }|x|>2 .\end{cases}
$$

Then $h$ is $A C L^{n}$ in $B=\left\{x \in \mathbf{R}^{n+1}\left|\frac{1}{2} \leq\right| x \mid<1\right\}$ since it is equal to $f \circ p$, where $p: B \rightarrow S^{n}$ is the radial projection (cf. Remark 6.4). In addition, $h$ is $C^{\mathbf{1}}$ in $C=\left\{x \in \mathbf{R}^{n+1}|2<| x \mid \leq 3\right\}$. Using Theorem 6.10 we define a sequence of $C^{\infty}$-maps $h_{i}^{\prime}: D \rightarrow \mathbf{R}^{n+1}-\{0\}, D=A \cup B \cup C$, such that $h_{i}^{\prime} \rightarrow h$ uniformly in $D$ and $D h_{i}^{\prime} \rightarrow D h$ in $L^{n}$-metric in each compact subset of $B$ or $C$. The maps $h_{i}: D \rightarrow S^{n}$ defined by $h_{i}$ $=h_{i}^{\prime} /\left|h_{i}^{\prime}\right|$ have the same properties since $\left|h_{i}^{\prime}\right| \rightarrow 1$ uniformly in $D$ and $D\left|h_{i}^{\prime}\right| \rightarrow 0$ in $L^{n}$-metric in each compact subset of $B$ or $C$. Hence there is a subsequence $h_{i_{j}}$ such that $D\left(h_{i_{j}} \mid S_{r}\right) \rightarrow D\left(h \mid S_{r}\right)$ in $L^{n}$-metric for almost all $r$ with $\frac{1}{2}<r<1$ or $2<r<3$. This completes the proof $\mathrm{n}$ the general case.

\section{Quasiconformal maps}

This section is devoted to the definition and study of some basic properties of quasiconformal maps of manifolds. Manifolds are assumed to be of dimension $\geq 2$.

7.1. Definition. Let $M$ and $N$ be Riemannian manifolds of dimension $n$. A homeomorphism $f: M \rightarrow N$ is called a quasiconformal map if there is a constant $K$ such that

$$
\frac{1}{K} M_{n}(\Gamma) \leq M_{n}\left(\Gamma^{\prime}\right) \leq K M_{n}(\Gamma)
$$

for each family $\Gamma$ of curves in $M$ and its image $\Gamma^{\prime}=f(\Gamma)$. In particular, if (7.1) is satisfied, $f$ is called $K$-quasiconformal.

A continuous map $f: M \rightarrow N$ is called quasiconformal into $N$ if there is an open Riemannian submanifold $N^{\prime} \subset N$ such that $f$ (int $\left.M\right)=N^{\prime}$ and $f \mid \operatorname{int} M$ : int $M \rightarrow N^{\prime}$ is quasiconformal.

7.2. Remark. If $f: M \rightarrow N$ is $K$-quasiconformal, then $f^{-1}: N \rightarrow M$, is also $K$-quasiconformal. If, in addition, $g: N \rightarrow L$ is $K^{\prime}$-quasiconformal then $g \circ f: M \rightarrow L$ is $K K^{\prime}$-quasiconformal.

7.3. Theorem. Let $N$ and $N^{\prime}$ be Riemannian manifolds of dimension $n$ and $f: N \rightarrow N^{\prime}$ a $C^{1}$-diffeomorphism. If the Riemannian metrics $g$ and $g^{\prime}$ of $N$ and $N^{\prime}$ satisfy 


$$
m^{2} g \leq f^{*} g^{\prime} \leq M^{2} g
$$

for some constants $0<m \leq M<\infty$, then $f$ is $(M / m)^{n}$-quasiconformal. A conformal map is 1-quasiconformal.

Proof: This follows immediately from Theorem 5.3.

7.4. Theorem. Let $M$ and $N$ be Riemannian manifolds of dimension $n$. Let $f: M \rightarrow N$ be a K-quasiconformal map. Then $f$ is differentiable almost everywhere and

$$
\|D f\|^{n} \leq K J_{f}
$$

at every point of differentiability.

Proof: If $M$ and $N$ are open sets in $\mathbf{R}^{n}$, the proof is given in Väisälä [1], Theorem 6.5. For the general case we may assume that $M$ and $N$ are diffeomorphic to open subsets $M^{\prime}$ and $N^{\prime}$ of $\mathbf{R}^{n}$, by means of $C^{1_{-}}$ diffeomorphisms $g: M \rightarrow M^{\prime}$ and $g^{\prime}: N \rightarrow N^{\prime}$. Then $g^{\prime} \circ f \circ g^{-1}$ is locally quasiconformal, hence differentiable a.e. This proves the first assertion.

In order to prove (7.2.) we assume that $f$ is differentiable at $x \in M$. Then $g$ and $g^{\prime}$ may be chosen so that they preserve the Riemannian metrics at $x$ and $f(x)$ and, if $M$ and $N$ are sufficiently small, they are $(1+\varepsilon)$-quasiconformal with $\varepsilon>0$ arbitrarily small. This completes the proof.

7.5. Theorem. Let $M$ and $N$ be Riemannian manifolds and $f: M \rightarrow N$ a quasiconformal map. Then

$$
\tau_{N}(f(E))=\int_{\boldsymbol{L}^{\prime}} J_{f} d \tau_{M}
$$

for each Borel set $E \subset M$. In particular, $\tau_{M}(E)=0$ implies $\tau_{N}(f(E))=0$. A Baire function $\varrho$ in $N$ is $\tau_{\mathrm{N}^{-}}$integrable if and only if $(\varrho \circ f) J_{f}$ is $\tau_{M^{-}}$ integrable, and

$$
\int_{N} \varrho d \tau_{N}=\int_{M}(\varrho \circ f) J_{f} d \tau_{M} .
$$

Proof: We first consider the case that $M$ and $N$ are open sets in $\mathbf{R}^{n}$. We apply a theorem of Lebesgue (Munroe [1], p. 285, Saks [1], p. 119) to the set function $\mu$ defined by $\mu(E)=\tau_{N}(f(E))$ for Borel sets $E \subset M$. It is easy to see that the derivative $\mu^{\prime}$ equals $J_{f}$ at each point where $f$ is differentiable. Hence (7.3) results if we know that $\tau_{M}(E)=0$ implies $\tau_{N}(f(E))=0$. But this is proved in Väisälä [1], Theorem 6.9.

If $M$ and $N$ are diffeomorphic to open subsets of $\mathbf{R}^{n}$, we prove (7.3) by means of Theorem 3.8, and remark for the general case that $M$ and $N$ 
may be covered by a countable family of coordinate neighbourhoods. By Lemma 3.4 (7.4) follows from (7.3).

7.6. Theorem. Let $M$ and $N$ be Riemannian manifolds of dimension $n$. Then every quasiconformal map $f: M \rightarrow N$ is $A C L^{n}$.

Proof: If $M$ and $N$ are open sets in $\mathbf{R}^{n}$, this follows from Theorem 6.5 in Väisälä [1] (cf. Remark 6.4). The general case is settled by means of Theorem 6.7.

7.7. Definition. Let $M$ and $N$ be Riemannian manifolds of dimension $n$ and $f: M \rightarrow N$ a quasiconformal map. We define two non-negative Baire functions $\lambda$ and $\delta$ in $M$ by

$$
\begin{aligned}
(\lambda(x))^{n} & =J_{f}(x), \\
\lambda(x)(1+\delta(x)) & =\|D f(x)\|, \quad x \in M .
\end{aligned}
$$

If $f$ is $K$-quasiconformal, then $(1+\delta)^{n} \leq K$ by (7.2).

7.8. Lemma. If $1 \leq(1+\delta)^{n} \leq K$, then $(1+\delta)^{n} \leq 1+n K \delta$.

Proof: This results immediately from the equation

$$
(1+\delta)^{n}-1=\delta\left((1+\delta)^{n-1}+\ldots+(1+\delta)+1\right) .
$$

7.9. Lemma. Let $M$ and $N$ be Riemannian manifolds of dimension $n$ and $f: M \rightarrow N$ a $K$-quasiconformal map. Then

$$
\left(\int_{E} \lambda(1+\delta) d \tau_{M}\right)^{n} \leq \tau_{M}(f(E)) \tau_{M}(E)^{n-2}\left(\tau_{M}(E)+n K \int_{E} \delta d \tau_{M}\right)
$$

and

$$
\tau_{N}(f(E)) \geq\left(\int_{E} \lambda(1+\delta) d \tau_{M} / \tau_{M}(E)\right)^{n}\left(\tau_{M}(E)-n K \int_{E} \delta d \tau_{M}\right)
$$

for each Borel set $E \subset M$.

Proof: By two applications of Hölder's inequality we obtain

$$
\begin{aligned}
\left(\int_{E} \lambda(1+\delta) d \tau_{M}\right)^{n} & \leq \int_{E} \lambda^{n} d \tau_{M}\left(\int_{E}(1+\delta)^{n /(n-1)} d \tau_{M}\right)^{n-1} \\
& \leq \tau_{N}(f(E)) \int_{E}(1+\delta)^{n} d \tau_{M} \cdot \tau_{M}(E)^{n-2}
\end{aligned}
$$

Hence (7.7) follows from Lemma 7.8 and (7.8) is an immediate consequence of $(7.7)$.

7.10. Lemma. Let $U$ and $V$ be domains in $\mathbf{R}^{n}$ and $f: U \rightarrow V$ a quasiconformal map. Then there is a constant $C$ such that for each $x \in C$, $r>0$, 


$$
M \leq C m
$$

with

$$
\begin{aligned}
& m=\min _{|y-x|=r}|f(y)-f(x)|, \\
& M=\max _{|y-x|=r}|f(y)-f(x)|,
\end{aligned}
$$

whenever $\bar{B}(x, r) \subset U, \bar{B}(f(x), M) \subset V$,

Proof: Let $\Gamma^{\prime}$ be the family of curves in $V$ which join $\{z|| z-f(x) \mid \leq m\}$ and $\{z|| z-f(x) \mid \geq M\}$. Let $\Gamma=f^{-1}\left(\Gamma^{\prime}\right)$. By Theorem $5.9 M_{n}(\Gamma) \geq \varkappa_{n}$ and by Theorem 5.10

$$
M_{n}\left(\Gamma^{\prime}\right)=\omega_{n}\left(\log \frac{M}{m}\right)^{1-n}
$$

Hence, if $f$ is $K$-quasiconformal,

$$
\varkappa_{n} \leq K \omega_{n}\left(\log \frac{M}{m}\right)^{1-n} .
$$

This proves the lemma with $C=\exp \left(\frac{K \omega_{n}}{\varkappa_{n}}\right)^{\frac{1}{1-n}}$.

\section{Dilatation}

In this section we define the dilatation of a quasiconformal map. The definition generalizes the concept of the complex dilatation in the twodimensional case (Lehto-Virtanen [1], p. 191). As a preliminary step we study the dilatation of a linear map.

8.1. Definition. Let $F$ be a euclidean space and $E$ a vector space of the same dimensionality. We denote by $\mathbf{I}(E, F)$ the set of invertible linear maps $u: E \rightarrow F$.

We establish an equivalence relation in the set of quadratic forms in $E$ by defining $Q$ and $Q^{\prime}$ related if $Q=\lambda Q^{\prime}$ for some $\lambda>0$. Then the set of equivalence classes of positive definite quadratic forms in $E$ is denoted by $\mathbf{Q}(E)$. If $\mu \in \mathbf{I}(E, F)$, then the quadratic form $Q$ defined by $Q(x)$ $=\|u(x)\|^{2}, \quad x \in E$; is positive definite. Its equivalence class in $\mathbf{Q}(E)$ is called the dilatation of $u$ and denoted by $\mu(u)$.

Let $\mathbf{C}(F)$ be the group of linear conformal maps $c: F \rightarrow F$. Then $\mathbf{C}(F)$ acts on $\mathbf{I}(E, F)$ by

$$
\begin{aligned}
& m: \mathbf{C}(F) \times \mathbf{I}(E, F) \rightarrow \mathbf{I}(E, F), \\
& m(c, u)=c \circ u .
\end{aligned}
$$


8.2. Theorem. Let $F$ be a euclidean space and $E$ a vector space of the same dimensionality. Then the map

$$
\mu: \mathbf{I}(E, F) \rightarrow \mathbf{Q}(E)
$$

determines a bijection of the quotient set of $\mathbf{I}(E, F)$ by $m$ onto $\mathbf{Q}(E)$.

Proof: Let $u, v \in \mathbf{I}(E, F)$ satisfy $u=c \circ v$ for some $c \in \mathbf{C}(F)$. Then $\|u(x)\|=\|c\| \cdot\|v(x)\|$ for each $x \in E$, and $\mu(u)=\mu(v)$. Conversely, if this holds, then clearly $u \circ v^{-1} \in \mathbf{C}(F)$. Since $\mu$ is surjective, this completes the proof.

8.3. Definition. Let $F$ be a euclidean space. Let $\mathbf{G L}(F)$ denote the group of invertible linear maps $a: F \rightarrow F$. Then, for each $a \in \mathbf{G L}(F)$, we define $\langle a\rangle=\|a\| \cdot\left\|a^{-1}\right\|$. It has the following properties:

a) $\langle a\rangle \geq 1$, for each $a \in \mathbf{G L}(F)$, and $\langle a\rangle=1$ if and only if $a \in \mathbf{C}(F)$,

b) $\langle a\rangle=\left\langle a^{-1}\right\rangle$, for each $a \in \mathbf{G L}(F)$,

c) $\langle a \circ b\rangle \leq\langle a\rangle\langle b\rangle$, for each $a, b \in \mathbf{G L}(F)$.

8.4. Theorem. Let $F$ be a euclidean space and $E$ a vector space of the same dimensionality. Then the function $d^{\prime}$ defined by;

$$
d^{\prime}(u, v)=\log \left\langle u \circ v^{-1}\right\rangle, \quad u, v \in \mathbf{I}(E, F),
$$

is a pseudometric in $\mathbf{I}(E, F)$. It determines a metric $d$ in $\mathbf{Q}(E)$ by

$$
d(\mu(u), \mu(v))=d^{\prime}(u, v), \quad u, v \in \mathbf{I}(E, F) .
$$

Proof: The first assertion results immediately from a) -c) of Definition 8.3. Since $\langle a \circ b\rangle=\langle a\rangle=\langle b \circ a\rangle$ if $\langle b\rangle=\left\langle b^{-1}\right\rangle=1$, it is clear that $d$ is well defined (Theorem 8.2). Finally, $d(\mu(u), \mu(v))=0$ implies $u \circ v^{-1} \in \mathbf{C}(F)$ and hence $\mu(u)=\mu(v)$. Thus $d$ is a metric and the proof is complete.

8.5. Remark. The metric $d$ in $\mathbf{Q}(E)$ defined in Theorem 8.4 is independent of the choice of the euclidean space $F$ as may be easily verified. It can also be seen that $\mathbf{Q}(E)$ has a natural manifold structure compatible with $d$, such that $\mu$ is differentiable.

8.6. Definition. Let $M$ and $N$ be Riemannian manifolds. Let $\mathbf{Q}(M)$ $=\mathbf{Q}(T M)$ be the tensor bundle of type $\mathbf{Q}$ over $M$ (cf. Lang [1], p. 49). Let $f: M \rightarrow N$ be a quasiconformal map. Then $D f(x) \in \mathbf{I}\left(T_{x} M, T_{f(x)} N\right)$ for a.e. $x \in M$ (cf. Theorem 7.5). The dilatation of $f$ is the section $\%$ of $\mathbf{Q}(M)$ defined by

$$
\varkappa(x)=\mu(D f(x))
$$

for a.e. $x \in M$. 
In the local case where $M=U$ is an open set in $\mathbf{R}^{n}$, the bundle $\mathbf{Q}(M)=U \times \mathbf{Q}\left(\mathbf{R}^{n}\right)$ is trivial and the dilatation may be represented by a $\operatorname{map} x: U \rightarrow \mathbf{Q}\left(\mathbf{R}^{n}\right)$.

The Riemannian metric of $M$ determines a canonical differentiable section of $\mathbf{Q}(M)$ which is denoted by $e$.

8.7. Theorem. Let $M$ and $N$ be Riemannian manifolds. If $f: M \rightarrow N$ is a quasiconformal map, then its dilatation $x$ is measurable. In particular, $d(\varkappa, e)$ is a Baire function in $M$.

Proof: We consider only the local case $M=U, N=V$ in $\mathbf{R}^{n}$; the general case is similar. The map $D f: U \rightarrow \mathbf{I}\left(\mathbf{R}^{n}, \mathbf{R}^{n}\right)$ is measurable by Theorem 2.10. Since $\mu$ is continuous, $x=\mu \circ D f$ is also measurable. The last assertion follows by the continuity of $d$.

8.8. Remark. To prove that $d(\varkappa, e)$ is a Baire function it is actually sufficient to consider only $I(E, F)$ and $d^{\prime}$. This is all that is needed in the sequel.

8.9. Lemma. The dilatation $x$ of a quasiconformal map $f: M \rightarrow N$ is related to $\delta$ by

$$
d(\varkappa, e) \leq n \log (1+\delta) \leq(n-1) d(x, e) \quad \text { a.e. }
$$

where $n$ denotes the dimension of $M$ and $N$.

Proof: Let $E$ be a euclidean space of dimension $n$ and $a: E \rightarrow E$ an invertible linear map. We denote $M=\|a\|$ and $m=\left\|a^{-1}\right\|^{-1}$. Then it is easy to see that

$$
m^{n-1} M \leq|\operatorname{det} a| \leq m M^{n-1} .
$$

On the other hand $M=\langle a\rangle m$ and $M^{n}=|\operatorname{det} a|(1+\delta)^{n}$ by Definitions 8.3 and 7.7. Combining these equations with (8.2) we obtain (8.1).

\section{Part II}

In this second part of the paper we generalize certain results of Teichmüller, Wittich and Belinskij for quasiconformal maps in higher dimensional spaces. We consider a quasiconformal map $f: U \rightarrow V$, where $U$ and $V$ are open submanifolds of $\mathbf{R}^{n}, n \geq 2$. We assume for simplicity that $0 \in U$ and $f(0)=0 \in V$. Let $\delta$ be the Baire function defined in Definition 7.7. Then we prove:

Theorem. If the integral

$$
\int \frac{\delta(x)}{|x|^{n}} d \tau
$$


is convergent then the quotient

$$
\frac{|f(x)|}{|x|}
$$

has a finite, non-zero limit at $x=0$. In addition, the maps $f_{r}: S^{n-1} \rightarrow S^{n-1}$, $0<r \leq r_{0}$, defined by

$$
f_{r}(x)=\frac{f(r x)}{|f(r x)|}
$$

converge to the set $I$ of isometries $g: S^{n-1} \rightarrow S^{n-1}$ in the following sense

$$
\lim _{r \rightarrow 0} \inf _{g \in I} \sup _{x \in S^{n-1}}\left|g(x)-f_{r}(x)\right|=0 .
$$

This theorem follows from Theorems 9.9 and 10.9. In the case $n=2$ it was shown by Belinskij (cf. Lehto-Virtanen [1], p. 239) that the maps $f_{r}$ converge to an isometry. This implies that $f$ is differentiable at $x=0$ (ibid., p. 243). We do not know whether this is true for $n>2$.

By Lemma 8.9 the convergence of the integral (*) is equivalent to the convergence of the following integral

$$
\int \frac{d(\varkappa, e)}{r^{n}} d \tau,
$$

where $r=|x|, \quad e \in \mathbf{Q}\left(\mathbf{R}^{n}\right)$ is the class of the canonical euclidean metric in $\mathbf{R}^{n}$ and $x$ is the dilatation of $f$ (Definition 8.6) considered as a measurable map $x: U \rightarrow \mathbf{Q}\left(\mathbf{R}^{n}\right)$. The integral (**) is meaningful also if $e$ is not the canonical class. Hence it gives a condition under which the theorem may be applied after a change of metric in $U$. In particular, it shows that the theorem is applicable if $x$ is Hölder-continuous.

Convention. In the rest of this paper the dimension $n \geq 2$ will be kept fixed. The Lebesgue measure of any $n$-dimensional manifold will be denoted by $\tau$ and the measure of manifolds of lower dimension by $\sigma$ and $\mu$.

\section{Radial convergence}

9.1. Definition. Let $S=\left\{x \in \mathbf{R}^{n}|| x \mid=r_{0}\right\}$ be the sphere of total measure one, i.e. $\omega_{n} r_{0}^{n-1}=1$. Let $L$ denote the product manifold $S \times \overline{\mathbf{R}}^{+}$. A map $\varphi: L \rightarrow \bar{B}-\{0\}, B=\left\{x \in \mathbf{R}^{n}|| x \mid<1\right\}$ is defined by

$$
\varphi(x, t)=x r_{0}^{-1} e^{-t / r_{0}}, \quad x \in S: t \in \overline{\mathbf{R}}^{+} .
$$

9.2. Theorem. The map $p: L \rightarrow \bar{B}-\{0\}$ is conformal. A quasiconformal map $f$ of $L$ into itself is proper, i.e., the preimage $f^{-1}(C)$ of each 
compact set $C \subset L$ is compact, if and only if there is a quasiconformal map $g$ of $\bar{B}$ into itself such that $\varphi \circ f=g \circ \varphi$ and $g(0)=0$.

Proof: The conformality of $\varphi$ is easily verified by direct calculation. Hence $g^{\prime}=\varphi \circ f \circ \varphi^{-1}$ is a quasiconformal map of $\bar{B}-\{0\}$ into itself. If $f$ is proper, $g^{\prime}$ may be extended to a continuous map $g: \bar{B} \rightarrow \bar{B}$ by setting $g(0)=0$. Since the family of all curves therough the origin has zero module, it is clear that $g$ is quasiconformal. Conversely, if there is a continuous map $g: \bar{B} \rightarrow \bar{B}$ satisfying $\varphi \circ f=g \circ \varphi$ and $g(0)=0$, it is immediate that $f$ is proper. This completes the proof.

9.3. Notation. Let $f$ be a proper $K$-quasiconformal map of $L$ into itself. Since $L$ is the product $S \times \overline{\mathbf{R}}^{+}$, we may represent $f$ by a pair of continuous map $g: L \rightarrow S$ and $h: L \rightarrow \mathbf{R}$. The set $S \times\{t\} \subset L$ is denoted by $S_{t}=S(t)$, for $t \geq 0$. We define continuous maps $g_{t}: S \rightarrow S$ and $h_{t}: S \rightarrow \mathbf{R}$ for $t \geq 0$ by

$$
g_{t}(x)=g(x, t), h_{l}(x)=h(x, t), \quad x \in S .
$$

We denote the oscillation of $h_{t}$ by $\Delta(t)$.

Let $x \in S(t)$ be a point at which $f$ has a non-zero derivative. Then the angle between $D f\left(T_{x} S(t)\right)$ and $T_{f(x)} S(h(x))$ is denoted by $\vartheta$. These notations are retained in the rest of this paper.

\subsection{Lemma.}

$$
\int_{S} J\left(g_{t}\right) d \sigma \geq 1
$$

for almost every $t \geq 0$.

Proof: By Theorem $6.9 g_{t}$ is $A C L^{n}$ for almost every $t \geq 0$. Hence, it is sufficient to show that $g_{t}$ is homotopic to a diffeomorphism, by Theorem 6.13.

By Theorem 9.2 there is a quasiconformal map $f_{1}$ of $\bar{B}$ into itself such that $\varphi \circ f=f_{1} \circ \varphi$ and $f_{1}(0)=0$. If $f_{1}$ is differentiable at the origin, with non-zero derivative, then $g_{t}$ tends to a diffeomorphism of $S$ onto itself as $t \rightarrow \infty$. Otherwise we replace $f_{1}$ by the map $f_{1}(x+a)-f_{1}(a)$ where $a$ is a point at which $f_{1}$ is differentiable. If $a$ is chosen sufficiently close to the origin, then $g_{t}$ is changed homotopically and the proof is concluded as above.

9.5. Lemma. There is a constant $A$ such that

$$
\int_{S(t)} J_{f}|\cos \vartheta| d \sigma \geq 1-A \int_{S(t)} \delta d \sigma
$$

for almost every $t \geq 0$.

Proof: By Lemma 9.4 


$$
\int_{S(t)} \lambda^{n-1}(1+\delta)^{n-1}|\cos \vartheta| d \sigma \geq 1
$$

for almost every $t \geq 0$. Thus by Hölder's inequality

$$
1 \leq \int_{S(t)}(1+\delta)^{n^{2}-n} d \sigma\left(\int_{S(t)} \lambda^{n}|\cos \vartheta|^{n /(n-1)} d \sigma\right)^{n-1} .
$$

On the other hand $(1+\delta)^{n} \leq K$, and hence

$$
\int_{S(t)}(1+\delta)^{n^{2}-n} d \sigma \leq 1+n(n-1) K^{n-1} \int_{S(t)} \delta d \sigma
$$

by Lemma 7.8. Thus

and finally

$$
\left(\int_{S(t)}(1+\delta)^{n^{2}-n} d \sigma\right)^{\frac{1}{n-1}} \leq 1+n K^{n-1} \int_{S(t)} \delta d \sigma
$$

$$
\int_{S(t)} J_{f}|\cos \vartheta| d \sigma \geq 1-n K^{n-1} \int_{S(t)} \delta d \sigma
$$

9.6. Lemma. There is a constant $B>0$ such that

$$
\int_{S(t)} J_{f}|\sin \vartheta|^{n} d \sigma \geq B \Delta(t)^{n}
$$

for almost every $t \geq 0$.

Proof: By Theorem 6.9 $h_{t}$ is $A C L^{n}$ for almost every $t \geq 0$. Let $y_{0}$ and $y_{1}$ be points where $h_{t}$ attains its maximal and minimal values, respectively. Let $\Gamma$ denote the family of curves $\gamma$ in $S_{t}$ joining $\left(y_{0}, t\right)$ and $\left(y_{1}, t\right)$, such that $h \circ \gamma$ is absolutely continuous and $D(h \circ \gamma)$ $=D h \circ D \gamma$. If $h_{t}$ is $A C L^{n}$, then $M_{n}(\Gamma)$ is greater than a positive constant $m$ depending only on $n$ by Corollary 5.8. We assume that this is the case.

Integrating $D h$ along $\gamma \in \Gamma$ we obtain

$$
\Delta(t)=h\left(y_{0}, t\right)-h\left(y_{1}, t\right) \leq \int_{\gamma} \lambda(1+\delta)|\sin \vartheta| d s .
$$

Thus $\lambda(1+\delta)|\sin \vartheta| \mid \Delta(t) \in F(\Gamma)$ and

$$
\int_{s(t)}[\lambda(1+\delta)|\sin \vartheta| \mid \Delta(t)]^{n} d \sigma \geq m
$$

This proves the assertion with $B=m / K$. 
9.7. Lemma. Let $E=L\left(t, t^{\prime}\right), 0 \leq t<t^{\prime}$, denote the set $S \times\left[t, t^{\prime}\right] \subset L$. Then

$$
\int_{E} J_{f} d \tau \leq t^{\prime}-t+n K \int_{E} \delta d \tau+2\left(\Delta(t)+\Delta\left(t^{\prime}\right)\right) .
$$

Proof: Let $d$ be the distance between $h\left(S_{t}\right)$ and $h\left(S_{t^{\prime}}\right)$. Then by (7.3)

$$
d \leq \int_{E} J_{f} d \tau \leq d+\Delta(t)+\Delta\left(t^{\prime}\right)
$$

It is obviously sufficient to consider the case $d>\Delta(t)+\Delta\left(t^{\prime}\right)$.

Let $\gamma_{x}, \quad x \in S$, denote the curve $\gamma_{x}(s)=(x, s), s \in\left[t, t^{\prime}\right]$. Then

$$
\int_{\gamma_{x}} \lambda(1+\delta) d s \geq d
$$

for almost every $x \in S$. Integrating over $S$ we obtain

$$
\int_{E} \lambda(1+\delta) d \tau \geq d
$$

Hence, by (7.7) and (9.2)

$$
d^{n} \leq\left(d+\Delta(t)+\Delta\left(t^{\prime}\right)\right)\left(t^{\prime}-t+n K \int_{E} \delta d \tau\right)\left(t^{\prime}-t\right)^{n-2}
$$

Since (9.1) follows from (9.2) if $d<t^{\prime}-t$, we may assume that $t^{\prime}-t \leq d$, whence

$$
d-\Delta(t)-\Delta\left(t^{\prime}\right) \leq t^{\prime}-t+n K \int_{E} \delta d \tau
$$

by (9.3). Combining this with (9.2) we obtain (9.1).

9.8. Lemma. A proper quasiconformal map $f$ of $L$ into itself is uniformly continuous.

Proof: Let $g$ be a quasiconformal map of $\bar{B}$ into itself satisfying $\varphi \circ f=g \circ \varphi$ and $g(0)=0$. Let $C$ be chosen as in Lemma 7.10 for $g$.

We choose positive numbers $a$ and $b$ such that

$$
g(B(2 a)) \subset B(b), B(3 b) \subset g(B) .
$$

Let $x \in \mathbf{R}^{n}$ be a point with $|x|=r<a$. Then by Lemma 7.10

$$
g(B(x, r)) \subset B\left(y, C r^{\prime}\right),
$$


where $y=g(x), \quad r^{\prime}=|y|$. Let $z$ be a point with $|z-x|=\delta<r$ and let $\delta^{\prime}$ denote $|g(z)-y|$. Then

$$
B\left(y, \delta^{\prime} / C\right) \subset g(B(x, \delta))
$$

by Lemma 7.10 again.

Let $\Gamma^{\prime}$ be the family of curves in $g(B)$ joining $S\left(y, C r^{\prime}\right)$ and $S\left(y, \delta^{\prime} / C\right)$. We denote $f^{-1}\left(\Gamma^{\prime}\right)$ by $\Gamma$. Then

and

$$
M_{n}(\Gamma) \leq \omega_{n}\left(\log \frac{r}{\delta}\right)^{1-n}
$$

$$
M_{n}\left(\Gamma^{\prime}\right)=\omega_{n}\left(\log \frac{C^{2} r^{\prime}}{\delta^{\prime}}\right)^{1-n}
$$

by Theorem 5.10. Hence

$$
K \omega_{n}\left(\log \frac{r}{\delta}\right)^{1-n} \geq \omega_{n}\left(\log \frac{C^{2} r^{\prime}}{\delta^{\prime}}\right)^{1-n}
$$

if $g$ is $K$-quasiconformal. Finally, this implies

$$
\frac{\delta^{\prime}}{r^{\prime}} \leq C^{2}\left(\frac{\delta}{r}\right)^{\alpha}, \quad \alpha=K^{\frac{1}{1-n}} .
$$

Passing over to $L$ by $\varphi$ we find that $f$ is, in fact, uniformly Höldercontinuous in $\varphi^{-1}(B(a))$. This completes the proof since $f$ is also uniformly continuous in the remaining compact set.

9.9. Theorem. If the integral $\int_{L} \delta d \tau$ is finite, then the functions $h_{t}-t$ in $S$ converge uniformly to a constant limit function as $t \rightarrow \infty$.

Proof: Since $2 \sin \vartheta / 2 \geq \sin \vartheta$ for $0 \leq \vartheta \leq \pi$, we have

$$
\cos \vartheta=1-2 \sin ^{2} \vartheta / 2 \leq 1-\frac{1}{2} \sin ^{n} \vartheta
$$

if $n \geq 2$. Taking Lemmas 9.5, 9.6 and 9.7 into account this yields

$$
\left.\frac{1}{2} B \int_{0}^{t} \Delta(t)^{n} d t \leq(A+n K) \int_{L(0, \mathrm{t})} \delta d \tau+2(J(0)-\lrcorner(t)\right)
$$

for all $t>0$. By Lemma 7.10 $\Delta(t)$ is bounded. Hence $\int \delta d \tau$ can be finite only if $\int \Delta(t)^{n} d t$ is finite. But $\Delta(t)$ is uniformly continuous by Lemma 9.8. Hence

$$
\lim _{t \rightarrow \infty} \Delta(t)=0 .
$$


This shows that if the functions $h_{t}-t$ converge, then the limit is constant and the convergence uniform.

To complete the proof we choose, for each $\varepsilon>0$, a $t_{0}>0$ such that

$$
\int_{L\left(t_{0}, t\right)} \delta d \tau<\varepsilon
$$

and $\Delta(t)<\varepsilon$ for $t \geq t_{0}$. Then by Lemma 9.7

$$
I=\int_{L\left(t_{0}, t\right)} J_{f} d \tau<t-t_{0}+n K \varepsilon+4 \varepsilon .
$$

On the other hand, Lemma 9.5 yields

$$
I>t-t_{0}-A \varepsilon
$$

Since the integral $I$ differs from $h(y, t)-h\left(y, t_{0}\right)$, for $y \in S$, by at most $\Delta\left(t_{0}\right)+\Delta(t)<2 \varepsilon$, we obtain

$$
\left|h(y, t)-t-h\left(y, t_{0}\right)+t_{0}\right|<(A+n K+6) \varepsilon
$$

for all $t \geq t_{0}$. Hence the functions $h_{t}-t$ converge and the proof is complete.

\section{Angular convergence}

10.1. Lemma. Let $I \subset \overline{\mathbf{R}}^{+}$be a closed interval of length $l$. If the oscillation of $h-t$ in $S \times I$ is smaller than $\varepsilon<l$, then

$$
\tau(f(E \times I)) \leq \sigma(E) l+(n+1) \varepsilon+n K \int_{S \times I} \delta d \tau,
$$

for each Borel set $E \subset S$.

Proof: Let $\gamma_{x}, x \in S$, denote the curve $\gamma_{i}(t)=(x, t), \quad t \in I$, in $S \times I$. Then we have

$$
\int_{\gamma_{x}} \lambda(1+\delta) d s \geq l-\varepsilon
$$

for almost every $x \in S$. Hence

$$
\int_{E \times I} \lambda(1+\delta) d \tau \geq(l-\varepsilon) \sigma(E) .
$$

By (7.8) we obtain 


$$
\begin{aligned}
\tau(f(E \times I)) & \geq\left(1-\frac{\varepsilon}{l}\right)^{n}\left(\sigma(E) l-n K \int_{E \times I} \delta d \tau\right) \\
& \geq \sigma(E) l-n \sigma(E) \varepsilon-n K \int_{E \times I} \delta d \tau .
\end{aligned}
$$

On the other hand, we have $\tau(f(S \times I)) \leq l+\varepsilon$. Hence, applying (10.2) to the set $F=S-E$, we obtain

$$
\tau(f(E \times I)) \leq l+\varepsilon-\sigma(F) l+n \sigma(F) \varepsilon+n K \int_{F \times I} \sigma d \tau
$$

and (10.1) follows.

10.2. Definition. Let $F$ be a $(n-2)$-dimensional linear subspace of $\mathbf{R}^{n}$. Let $\iota_{1}$ and $\varphi_{1}, \quad 0 \leq \varphi_{1}<2 \pi$, denote polar coordinates in the orthogonal complement $E$ of $F$. For each point $x \in \mathbf{R}^{n}$ let $y(x)$ and $z(x)$ be the components of $x$ in $E$ and $F$. Then the functions $\varrho, \varphi$ and $z$ in $\mathbf{R}^{n}$ defined by $\varrho=\varrho_{1} \circ y, \varphi=\varphi_{1} \circ y$, are called cylindrical coordinates with axis $F$. A point $x \in \mathbf{R}^{n}$ is also denoted by $(\varrho(x), \varphi(x)$, $z(x))$.

10.3. Notation. Let $a$ and $b$ be two distinct points of $S$ (Definition 9.1). Let $F$ denote the orthogonal complement of the plane spanned by $a$ and $b$, if $a \neq-b$. In case $a=-b, F$ may be any (n-2)-dimensional subspace of $\mathbf{R}^{n}$, orthogonal to $a$ and $b$. Let $\varrho, \varphi$ and $z$ be cylindrical coordinates with axis $F$, chosen so that $\varphi(a)=0,0<x=\varphi(b) \leq \pi$.

Let $r>0$ and $0 \leq \varphi<2 \pi$. The set $\{x \in S|| z(x) \mid \leq r, \varphi(x)=\varphi\}$ is denoted by $C_{\varphi}=C_{\varphi}(r)$ and its $(n-2)$-measure by $m=m(r)$. The union $\cup C_{\varphi}(r), 0 \leq \varphi \leq \alpha$, is denoted by $A(r)$ or, briefly, $A$.

10.4. Lemma. Let $\varrho$ be a Baire function in $A$. Then

$$
\int_{A} \varrho d \sigma=\int_{C_{0}} d \mu \int_{\gamma_{y}} \varrho d s
$$

where $\gamma_{y}, y \in C_{0}$, denotes the curve $\gamma_{y}(t)=(\varrho(y), t, z(y)), t \in[0, \alpha]$, in $A$.

Proof: Let $I=[0, a]$. Then the map $f: C_{0} \times I \rightarrow A$ defined by

$$
f(y, t)=(\varrho(y), t, z(y)), \quad y \in C_{0}, t \in I,
$$

is a diffeomorphism. Hence

$$
\int_{A} \varrho d \sigma=\int_{C_{0} \times I}(\varrho \circ f) J_{f} d \sigma=\int_{C_{0}} d \mu \int_{\gamma_{y}}(\varrho \circ f) J_{f} d s,
$$


where $\gamma_{y}^{\prime}, y \in C_{0}$, denotes the curve $\gamma_{y}^{\prime}(t)=(y, t), t \in I$, in $C_{0} \times I$. Since it is easy to see that

$$
\int_{\gamma_{y}^{\prime}}(\varrho \circ f) J_{f} d s=\int_{\gamma_{y}} \varrho d s
$$

this completes the proof.

10.5. Lemma. Let $I \subset \overline{\mathbf{R}}^{+}$be a closed interval of length $l$ and let $d$ denote the distance between $g\left(C_{0} \times I\right)$ and $g\left(C_{\alpha} \times I\right)$ in $S$. Then

$$
k^{n} \tau(f(A \times I)) \geq \sigma(A) l-n K \int_{A \times I} \delta d \tau
$$

where $k=d(a, b) / d$.

Proof: Let $A_{t}, t \in I$, denote the set $A \times\{t\} \subset A \times I$. If $f \mid S_{t}$ is $A C L^{n}$, then

$$
\int_{\lambda_{t}} \lambda(1+\delta) d \sigma \geq d m(r)
$$

by Lemma 10.4. Since this is the case for almost every $t \in I$ by Theorem 6.9 , we obtain

$$
\int_{A \times \mathbf{I}} \lambda(1+\delta) d \tau \geq d m l
$$

Hence, by (7.8),

$$
\tau(f(A \times I)) \geq(d m / \sigma(A))^{n}\left(\sigma(A) l-n K \int_{\boldsymbol{A} \times \mathbf{I}} \delta d \tau\right) .
$$

On the other hand $\sigma(A) \leq m d(a, b)$ and (10.3) follows.

10.6. Lemma. Let $X$ be a compact metric space and $a: X \rightarrow \mathbf{R} a$ continuous function. Then the sets $\{x \in X|| a(x) \mid<\varepsilon\}, \varepsilon>0$, form $a$ base for the neighbourhood system of the set $A=a^{-1}(0)$.

Proof: If the assertion does not hold, then for some neighbourhood $U$ of $A$ there is a sequence $\left(x_{i}\right), i=1,2, \ldots$, of points in $X-U$ such that $a\left(x_{i}\right) \rightarrow 0$. Since $X$ is compact, we may assume that $x_{i} \rightarrow x$ in $X-U$. This leads to a contradiction with $a(x)=0$.

10.7. Definition. Let $F$ be the set of all continuous maps $f: S \rightarrow S$ which are homotopic to a diffeomorphism. We define a metric $d$ in $F$ by

$$
d(f, g)=\sup _{x \in S} d(f(x), g(x)), \quad f, g \in F .
$$

The set of all isometric maps $f: S \rightarrow S$ is denoted by $I$. 
10.8. Lemma. The function $a: F \rightarrow \mathbf{R}$ defined by

$$
a(f)=\sup _{\boldsymbol{x}, y \in S}[d(f(x), f(y))-d(x, y)], \quad f \in F,
$$

is continuous and non-negative. If $f \in F$, then $a(f)=0$ if and only if $f$ is an isometry.

Proof: The continuity of $a$ is clear. Let $f \in F$ satisfy $a(f) \leq 0$, i.e., $d(f(x), f(y)) \leq d(x, y)$ for $x, y \in S$. Then $f$ maps each pair of opposite points in $S$ into a pair of opposite points, since otherwise $f$ is not surjective. But $f(-x)=-f(x)$ implies that $d(f(x), f(y)) \geq d(x, y)$ for $x, y \in S$ Hence $f$ must be an isometry.

10.9. Theorem. If the integral $\int_{\boldsymbol{L}} \delta d \tau$ is finite, then

as $t \rightarrow \infty$.

$$
d\left(g_{t}, I\right) \rightarrow 0
$$

Proof: The functions $g_{t}, t \geq 0$, form an equicontinuous family by Lemma 9.8. Hence the closure $X \subset F$ of the set $\left\{g_{t} \mid t \geq 0\right\}$ is compact by Ascoli's theorem (Kelley [1], p. 233). Then, by Lemmas 10.6 and 10.8, it suffices to show that $a\left(g_{t}\right) \rightarrow 0$ as $t \rightarrow \infty$.

Choose $\eta>0$. Since $f$ is uniformly continuous, we can find $r>0$ and $l>0$ such that the oscillation of $g$ is smaller than $\eta / 4$ in each set of the form $C_{\varphi}(r) \times I$ (Notation 10.3), where $I \subset \overline{\mathbf{R}}^{+}$is a closed interval of length $l$.

Let $a$ and $b$ be distinct points of $S$, let $I_{t}=[t, t+l]$ for $t \geq 0$, and let $\varepsilon_{t}$ be the oscillation of the function $h-t$ in $S \times I_{t}$. By Lemmas 10.1 and 10.5 we then obtain (cf. Notation 10.3) for large enough $t$

$$
k_{t}^{n}\left(\sigma(A) l+(n+1) \varepsilon_{t}+n K \int_{A \times I_{t}} \delta d \tau\right) \geq \sigma(A) l-n K \int_{A \times I_{t}} \delta d \tau,
$$

where

$$
k_{t} \leq d(a, b) /\left(d\left(g_{t}(a), g_{t}(b)\right)-\eta / 2\right) .
$$

Since $\varepsilon_{t} \rightarrow 0$ and $\int_{A \times I_{t}} \delta d \tau \rightarrow 0$ as $t \rightarrow \infty$, it follows that

Hence

$$
\liminf _{t \rightarrow \infty} k_{t} \geq 1 \text {. }
$$

if $t$ is large enough. In addition, (10.4) and hence (10.5) hold uniformly in $a$ and $b$ if $\sigma(A)$ is bounded away from zero, i.e. $d(a, b) \geq m$ for some $m>0$. Since the case where $d(a, b)<m$ may be taken care of by using the uniform continuity of $f$, the proof is complete. 


\section{References}

Bourbaki, N.: [1] Topologie générale, ch. 1-2, $3^{\mathrm{e}}$ ed., Act. Sci. Ind. 1142, Hermann, Paris, 1961.

Kelley, J. L.: [1] General Topology, D. Van Nostrand Co., Inc., Princeton, N. J., 1955.

LaNG, S.: [1] Introduction to Differentiable Manifolds, Interscience Publishers, New York, 1962.

Lehto, O. - Virtanen, K. I.: [1] Quasikonforme Abbildungen, Springer-Verlag, Berlin-Heidelberg-New York, 1965.

Munroe, M. E.: [1] Introduction to Measure and Integration, Addison-Wesley Publishing Co., Inc., Reading, Mass., 1953.

Riesz, F. - Sz.-Nagy, B.: [1] Leçons d'analyse fonctionnelle, Akadémiai Kiadó, Budapest, 1952 .

SAKs, S: [1] Theory of the Integral, $2^{\text {nd }}$ ed., Monografie matematyczne, Warszawa, 1937.

VäısÄLё J.: [1] On quasiconformal mappings in space, Ann. Acad. Sci. Fenn. 298, 1961. 